\title{
A Long-Term Mathematical Model for Mining Industries
}

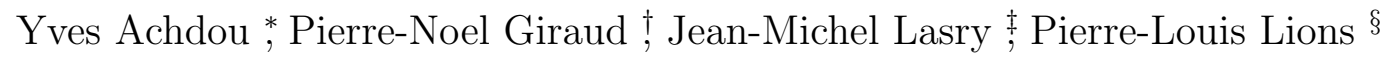

November 11, 2016

\begin{abstract}
A parcimonious long term model is proposed for a mining industry. Knowing the dynamics of the global reserve, the strategy of each production unit consists of an optimal control problem with two controls, first the flux invested into prospection and the building of new extraction facilities, second the production rate. In turn, the dynamics of the global reserve depends on the individual strategies of the producers, so the models leads to an equilibrium, which is described by low dimensional systems of partial differential equations. The dimensionality depends on the number of technologies that a mining producer can choose. In some cases, the systems may be reduced to a Hamilton-Jacobi equation which is degenerate at the boundary and whose right hand side may blow up at the boundary. A mathematical analysis is supplied. Then numerical simulations for models with one or two technologies are described. In particular, a numerical calibration of the model in order to fit the historical data is carried out.
\end{abstract}

\section{Introduction}

Mean field type models describing the asymptotic behavior of stochastic differential games (Nash equilibria) as the number of players tends to $+\infty$ have been introduced and termed mean field games in $[10,11,12]$. For brevity, the acronym $M F G$ will sometimes be used for mean field games. Since its introduction ten years ago, the topic has attracted the attention of many researchers, so many that it has become almost impossible to list all the references. The models have been applied to many areas such as economics, finance, social sciences and engineering. Examples of MFG models with applications in economics and social sciences are proposed in $[6,1]$. The first articles $[10,11,12]$ mostly dealt with the cases when each player is exposed to an independent source of risk (idiosynchratic risk). The case when there is a risk common to all players (in addition to the abovementionned idiosynchratic risks) is much more difficult and has been first studied by J-M. Lasry and P-L. Lions, in order to tackle analytically the questions raised in macro-economics by Krussel and Smith in [7] and previously investigated via numerical approaches. Such models lead to the so-called master equations, a term chosen for some second order partial differential equations set in a space of probability measures. The

\footnotetext{
*Univ. Paris Diderot, Sorbonne Paris Cité, Laboratoire Jacques-Louis Lions, UMR 7598, UPMC, CNRS, F-75205 Paris, France

${ }^{\dagger}$ CERNA, Mines ParisTech

${ }^{\ddagger}$ Univ. Paris Dauphine

${ }^{\S}$ Collège de France

${ }^{1}$ All the authors acknowledge the support of the Chaire "Finance et développement durable". The last three authors were partially supported by the Chaire "Économie, finance et gestion des commodités". The first author was partially supported by ANR projects ANR-12-MONU-0013 and ANR-16-CE40-0015-01.
} 
well-posedness of master equations has been discussed in the recent article [5] in some particular cases, together with the convergence as the number of players tends to $+\infty$. When there is common noise and when the space of states is finite (of cardinal $d$ ), the MFG master equations reduce to systems of hyperbolic equations in $\mathbb{R}^{d}$; some applications of this class of MFG have already been investigated in [8]. The specific model discussed hereafter will also belong to this class.

Mining industries have several specifities which are well taken into account by mean field games (MFG) models. The present work is devoted to the dynamics of mining industries on very long time periods and at an aggregate level, for which a MFG model will be proposed.

More precisely, we are interested in steady state MFG systems that lead to a good approximation of the so called cost curve and involve a quite parcimonious parametrization. The interest of this reduced parametric model is to lead to low dimensional MFG systems that can be solved numerically and tested by comparison with available historical data. The adequation of the model with historical observations a posteriori supports its validity.

The interest of the models proposed in the present paper in terms of economic analysis will be tackled in more details in another article in preparation [2], in which we shall analyze the qualitative and quantitative agreement of the model with the economic observations and the historical data. Therefore, after a short introduction on mining industries, we will focus on the mathematical and numerical aspects of our models and their calibration from historical data, and leave most of the economic interpretation for the other paper.

Our model is based on a counter-intuitive approximation, namely that the mining resources are inexhaustible. Of course, this is contradicted by elementary physics: the physical quantity of ores on the ground is finite and cannot be inexhaustible. But, even if we are interested in a model in the long run, we must keep in mind what "long run" means in economics: for us, ten decades are already quite a long run, and the data from mining industries suggest that within this time horizon, a large number of mineral resources may be considered as inexhaustible. A consequence of the approximation above is that the limitations on the fluxes of newly available reserves are only caused by the fluxes of investments into prospection. In [2], we will give more details on the validity of this approximation.

The latter leads us to make another important structural choice: our model will be stationary up to a scale factor. This choice can also be justified by economic arguments that we will supply in [2]. Here, we limit ourselves to saying that the assumption on stationarity will be a posteriori justified by observing that our model satisfactorily agrees with historical data. Moreover, the hypothesis of stationarity will partly explain the simplicity and parcimony of the model.

In mining industries, the capital has essentially two functions: prospection for new deposits, and construction of new extraction facilities, which create a production system characterized by: the available reserve of ore, the annual production capacity, the operational cost of extraction. Production decreases the available reserve, and ceases when the reserve is drained. On the long term, mining industries rely on a continuous flux of investments on prospection and building extraction capacities.

The present model is adapted to the long-term analysis (on several decades) of the dynamics of the prices of commodities on global markets. We shall neglect short-term phenomenons and regional disparities.

Mining producers are distinguished from each other by their prospection costs, their costs for constructing facilities, and their operational costs of extraction. We shall however simplify the model by supposing that the costs of prospection and of building facilities only depend on the size of the available reserve possessed by the producer. We shall also assume that the operational cost of production are constant in time and can take only a small number $n$ of different values. 
In the language of mining industry, this amounts to saying that the cost curve is piecewise constant. We shall see that the simplest model, namely with $n=1$, is already in good adequation with the historical data on four decades.

On the mathematical and numerical viewpoint, the model leads to a MFG system in $n$ dimensions, with specific difficulties related in particular to boundary conditions, and which is equivalent to a Hamilton-Jacobi-Bellman equation in the absence of economic frictions.

\section{Mathematical models}

\subsection{The simpler model: a closed industry with a single technology}

\subsubsection{The main economic assumptions}

We consider a mining industry composed of production units in competition. In this first model, only the existing production units can prospect for new resource and build new production facilities.

The main assumptions are as follows:

- The term reserves means reserves immediately available for production.

- Each production unit has two controls: the production rate and the investments in prospecting for new reserves/building new production structures. In what follows, we will not make the distinction between prospecting for new reserves and building new production facilities. Both activities will be termed prospection.

- There is only one type of production unit, i.e. there is only one technology; thus all units have the same prospection and production costs.

- Scale invariance: to stay as simple as possible, we make the following two assumptions which will imply that the dynamics of the industry is independent of the size of the production units, i.e. the size of their reserves:

- the production capacity of a given production unit is proportional to its reserve, with the same proportionality factor for all geographic locations, all times and all production units: there exists a constant $0<k \leq 1$ such that the maximal capacity of extraction of any producer during $d t$ is $k \rho d t$ if its reserve is $\rho$.

- There exists an exogeneous function $\phi$, identical for all geographic locations, all times and all production units, such that, for a given producer whose reserve is $\rho$, an investment into prospection of $\alpha d t$ during $d t$ increases its reserve by $d \rho=\rho \phi(\alpha / \rho) d t$. Assuming the latter instead of more general law of the form $d \rho=\psi(\alpha, \rho) d t$ amounts to supposing that what really matters is the ratio of the flux of investment to the available reserve, whatever the size of the production unit may be.

The two assumptions above make it unnecessary to distinguish between the sizes of the operators which compose the industry. They will allow us to consider that all production units have the same unitary reserve (this amounts to splitting production units so that they all have unitary reserve). Hence, the model will be built on the utility function of a production unit whose reserve is of 1 .

More precisely, 
- The total reserve will be noted $R(t) \in \mathbb{R}_{+}$. With the convention above, $R(t)$ can also be viewed as the quantity of production units.

- Let $k, 0<k \leq 1$ be the production capacity of a single production unit (with reserve 1 ).

- Let $c>0$ be the unitary production cost, i.e. the production cost of a unit of ore.

- Each production unit can invest into prospection. The flux invested into prospection by a single unit is $\alpha d t$ ( $\alpha$ is a control parameter). An investment rate of $\alpha$ results in increasing the reserves at a rate $\phi(\alpha)$, where $\phi$ is an increasing and concave function on $\mathbb{R}_{+}$such that $\phi(0)=0$. For example, one may choose $\phi(\alpha)=\sqrt{\alpha}$. Translated into the equivalent model where production units may have different sizes, this means that if the reserve of a production unit is $\rho$, and the flux spent into investment during $d t$ is $\alpha$, then the reserve will be increased by $\rho \phi(\alpha / \rho) d t$.

- The other control parameter is the production rate $\beta$ of a single unit, with $0 \leq \beta \leq k$.

- The discount factor of the expected income is $r>0$.

Systemic risk: an exogeneous demand function A source of common noise is the exogeneous demand function: it is assumed that the demand function has the form $D(X, p)=X \tilde{D}(p)$ where $p$ is the unitary price of ore and $X$ is a random positive parameter standing for the state of the economy. The function $\tilde{D}$ is defined on $(0,+\infty)$, takes nonnegative values, is nonincreasing with respect to $p>0$ and decreasing in the interval $\tilde{D}^{-1}(0,+\infty)$. It tends to 0 at $+\infty$. For example, one can take $\tilde{D}(p)=(1-\epsilon p)_{+}$where $\epsilon$ is a positive parameter, or $\tilde{D}(p)=p^{-s}$ where $s$ is a positive exponent.

The dynamics of $X$ is assumed to be of the form

$$
d X_{t}=X_{t}\left(b d t+\sigma d W_{t}\right)
$$

where $W_{t}$ is a standard Brownian motion and $b$ is the average growth rate of the economy, and the volatility $\sigma$ is a nonnegative constant (for simplicity).

The noise induced by $X_{t}$ is felt by all the agents, i.e. the production units: this is why $X_{t}$ is refered to as a common noise in the theory of mean field games, see $[13,5]$. In different kind of MFG models, the agents are affected by idiosynchratic noises (an independent stochastic process for each agent), to which a common noise may or may not be added.

\subsubsection{The strategy of the production units}

We are going to propose a model of mean field games leading to some master equation in the terminology introduced in [13], see also [5]. The solution $u(R, X)$ of this equation is the expected discounted value of one unit of extracted ore, or equivalently the value of a production unit. It will be obtained as the value function associated to an optimal control problem in $\alpha$ and $\beta$, that each production unit will solve knowing the dynamics of the global reserve. Since the latter in turn depends on $u$, the model will lead to a mean field equilibrium.

Note that the unknown of the master equation is generally a function whose arguments include a measure on the state space standing for the distribution of the agents and possibly other exogeneous parameters. Hence the master equation is generally infinite dimensional. This is a serious difficulty, which has been handled in $[13,5]$. Yet, in the special case that we are dealing with, all the production units are in the same state, because there is only one technology. Therefore, the previously mentioned distribution is a Dirac mass whose intensity is $R$ (the number 
of production units). The master equation will thus be bidimensional with the two variables $R$ and $X$.

In $\S 4$, we are going to discuss a model with two possible technologies: in this case, the distribution of the production units will be a sum of two Dirac masses. The master equation will then be a partial differential equation with three variables, the numbers $R_{1}$ and $R_{2}$ of the production units of types 1 and 2 respectively, and the state of the economy $X$.

Recall that $r$ is the discount rate.

When a production unit produces $q$ units of ore, its costs can be shared into two parts:

- a production cost of $q c$

- a decrease of the reserves of $q$, which costs $q u(R, X)$,

so the total cost is $q c+q u(R, X)$ while the income is $p q$. Therefore, the following inequality must hold:

$$
p \geq c+u(R, X) .
$$

If $p=c+u(R, X)$, it is indifferent for a production unit to produce or not.

Fixing the price $p$ and the global production $Q$ knowing $u(R, X)$ The unit price $p$ of ore and the global production $Q$ can be found by using (2) and matching offer and demand.

Let $P^{*}(R, X, u)$ and $Q^{*}(R, X, u)$ be respectively the price and global production functions. The cash income for a unit of ore produced by the industry is

$$
g(R, X, u)=P^{*}(R, X, u)-c .
$$

There are thus two cases:

1. The industry produces at full capacity when $p>u+c$.

The total production is $Q=k R$. Matching offer and demand yields $k R=D(X, p)$, i.e $P^{*}(R, X, u)=\tilde{D}^{-1}(k R / X)$. The inequality $P^{*}(R, X, u)>u+c$ is then equivalent to $\tilde{D}(u+c)>k R / X$. In this regime, $g(R, X, u)=\tilde{D}^{-1}(k R / X)-c$.

2. The industry has a partial production when $u+c=p$. The total production $Q$ is obtained by matching offer and demand: $Q=D(X, u+c)$. In this regime, $0 \leq \tilde{D}(u+c)<$ $k R / X$ and $g(R, X, u)=u$.

To summarize, setting $\tilde{D}^{-1}(z)=-\infty$ if $z>\lim _{p \rightarrow 0^{+}} \tilde{D}(p)$, and $\tilde{D}(p)=+\infty$ is $p<0$,

$$
\begin{aligned}
P^{*}(R, X, u) & =\max \left(\tilde{D}^{-1}\left(\frac{k R}{X}\right), u+c\right), \\
\frac{Q^{*}(R, X, u)}{X} & =\min \left(\tilde{D}(u+c), \frac{k R}{X}\right), \\
g(R, X, u) & =\max \left(\tilde{D}^{-1}\left(\frac{k R}{X}\right)-c, u\right) .
\end{aligned}
$$

As always in mean field games, we are interested in finding an equilibrium where

1. each production unit chooses its strategy given the dynamics of some aggregate quantities, here $R_{t}$ (and $X_{t}$ which is exogeneous)

2. conversely, the evolution of the aggregate quantity $R_{t}$ is deduced from the previously mentioned individual optimal controls. 
The optimal strategy of a production unit A production unit using a policy $(\alpha, \beta)$ during a small time interval of duration $d t$ increase its reserve by $(\phi(\alpha)-\beta) d t$. Since all units are identical, we can assume that they all use the same strategy, (even if there may be situations of indifference), that is obtained by solving the following optimization problem, knowing the dynamics of the aggregate quantities $R$ and $X$ :

$$
u(R, X)=(1-r d t) \max _{\alpha>0,0 \leq \beta \leq k} \mathbb{E}\left(\begin{array}{l}
(\beta g(R, X, u)-\alpha) d t \\
+(1+\phi(\alpha) d t-\beta d t) u(R+d R, X+d X)
\end{array}\right) .
$$

A first order expansion yields

$$
\begin{aligned}
0= & -r u(R, X)+k(g(R, X, u)-u(R, X))+\max _{\alpha}(\phi(\alpha) u(R, X)-\alpha) \\
& +\partial_{R} u \frac{d R}{d t}+\left(b X \partial_{X} u+\frac{1}{2} \sigma^{2} X^{2} \partial_{X X} u\right),
\end{aligned}
$$

where the optimal $\beta$ has been given by

$$
\begin{aligned}
\beta^{*}=k & \text { if } g(R, X, u)-u>0, \\
\beta^{*}=\frac{D(X, u+c)}{R} & \text { if } g(R, X, u)-u=0 .
\end{aligned}
$$

While (8) is unambiguous, (9) needs to be explained: in the regime when $g(R, X, u)-u=0$, the units are indifferent to producing or not, hence, $\beta^{*}$ is not really characterized. To fix $\beta^{*}$, we have arbitrarily imposed that all units behave the same way, so in $(9), \beta^{*}$ is obtained by matching the global offer and the demand: $R \beta^{*}=D(X, u+c)$. This choice does not affect $(7)$, because $\beta$ is multiplied by $g(R, X, u)-u=0$.

At the equilibrium, if $\alpha^{*}$ is the optimal value of $\alpha$ and if $Q^{*}(R, X, u)$ is the demand $Q^{*}(R, X, u)=R \beta^{*}$, then the aggregate quantity $R$ evolves as follows:

$$
d R=\left(R \phi\left(\alpha^{*}\right)-Q^{*}(R, X, u)\right) d t .
$$

To summarize, we get the partial differential equation

$$
-r u+k(g(\cdot, u)-u)-Q^{*}(\cdot, u) \partial_{R} u+\partial_{R}\left(R \max _{\alpha}(u \phi(\alpha)-\alpha)\right)+b X \partial_{X} u+\frac{1}{2} \sigma^{2} X^{2} \partial_{X X} u=0 .
$$

We are interested in nonnegative solutions of (11).

\subsubsection{Reduced forms of (11)}

Homogeneity: a reduced variable Observe that $g$ and $\tilde{Q}^{*}=Q^{*} / X$ only depend on $R / X$ and $u$. Introduce the reduced variable $y=R / X$; looking for a nonnegative solution of (11) of the form $u(R, X)=v(y)$, we obtain the second order differential equation:

$$
-r v+k(g(y, v)-v)-\tilde{Q}^{*}(y, v) v^{\prime}+\frac{d}{d y}\left(y \max _{\alpha}(v \phi(\alpha)-\alpha)\right)+\left(\sigma^{2}-b\right) y v^{\prime}+\frac{\sigma^{2} y^{2}}{2} v^{\prime \prime}=0 .
$$

Using (5) and (6), (12) becomes

$$
\begin{aligned}
0= & -r v+k 1_{\left\{\tilde{D}^{-1}(k y) \geq v+c\right\}}\left(\tilde{D}^{-1}(k y)-c-v-y v^{\prime}\right)-1_{\{\tilde{D}(v+c)<k y\}} \tilde{D}(v+c) v^{\prime} \\
& +\frac{d}{d y}\left(y \max _{\alpha}(v \phi(\alpha)-\alpha)\right)+\left(\sigma^{2}-b\right) y v^{\prime}+\frac{\sigma^{2} y^{2}}{2} v^{\prime \prime} .
\end{aligned}
$$

We will see in the paragraph below that (13) is a viscous conservation law with a flux function which is continuous but not smooth: the lack of smoothness occurs at the transition between two regimes, which respectively correspond to full and partial capacity productions. We are interested in nonnegative solutions of (13). 
A Hamilton-Jacobi equation For what follows, it is useful to notice that for any $M>0$, the function defined on $\mathbb{R}_{+} \times \mathbb{R}_{+}$by

$$
y \mapsto 1_{\{\tilde{D}(v+c)<k y\}} \int_{v+c}^{M} \tilde{D}(z) d z-1_{\{\tilde{D}(v+c) \geq k y\}}\left(k y\left(v+c-\tilde{D}^{-1}(k y)\right)-\int_{D^{-1}(k y)}^{M} \tilde{D}(z) d z\right)
$$

is a primitive of

$$
y \mapsto k 1_{\left\{\tilde{D}^{-1}(k y) \geq v+c\right\}}\left(\tilde{D}^{-1}(k y)-c-v-y v^{\prime}\right)-1_{\{\tilde{D}(v+c)<k y\}} \tilde{D}(v+c) v^{\prime} .
$$

Let the Hamiltonian $H_{1}(y, v)$ be the continuous function defined on $\mathbb{R}_{+} \times \mathbb{R}_{+}$by

$$
\begin{aligned}
& H_{1}(y, v) \\
= & -1_{\{\tilde{D}(v+c)<k y\}} \int_{v+c}^{M} \tilde{D}(z) d z+1_{\{\tilde{D}(v+c) \geq k y\}}\left(k y\left(v+c-\tilde{D}^{-1}(k y)\right)-\int_{\tilde{D}^{-1}(k y)}^{M} \tilde{D}(z) d z\right) .
\end{aligned}
$$

It can be checked that $H$ is continuous on $\mathbb{R}_{+} \times \mathbb{R}_{+}$, and even $\mathcal{C}^{1}$ if $\tilde{D}$ is $\mathcal{C}^{1}$. Note the following monotonicity property:

$$
\left(H_{1, v}(y, v)-H_{1, v}(z, w)\right)(v-w)-\left(H_{1, y}(y, v)-H_{1, y}(z, w)\right)(y-z) \leq 0 .
$$

It is also useful to introduce the second Hamiltonian

$$
H_{2}(y, v)=-y \max _{\alpha \geq 0}(v \phi(\alpha)-\alpha),
$$

which also satisfies (16). Finally, let the global Hamiltonian $H(y, v)$ be defined by

$$
H(y, v)=H_{1}(y, v)+H_{2}(y, v) .
$$

Then (13) admits the conservative form

$$
(b-r) v-\frac{d}{d y}(H(y, v))+\frac{d}{d y}\left(\frac{\sigma^{2} y^{2}}{2} \frac{d v}{d y}-b y v\right)=0 .
$$

Consider now the Hamilton-Jacobi equation

$$
(r-b) V+b y V^{\prime}+H\left(y, V^{\prime}\right)-\frac{\sigma^{2} y^{2}}{2} V^{\prime \prime}=0 .
$$

By deriving (19), we observe that if $V$ is a nondecreasing solution to (19), then $v=V^{\prime}$ is a nonnegative solution to (12).

The connection between the master equation (13) and the Hamilton-Jacobi equation (19) is a typical instance of potential games in which the equilibria can be found as the solutions of a central planner problem. In a work in progress, [2], we will put the focus on economic aspects and discuss the central planner problem connected with the present mean field game. To be more explicit on the analytical treatment of this connection, we propose in Appendix A a MFG formulation of Lucas-Prescott celebrated model, see [14]: in this case, we show how the MFG master equation leads to a Hamilton-Jacobi equation, which is precisely the Lucas-Prescott equation. The reader will then be able to compare the two ways to obtain the Lucas-Prescott equation, either via the MFG theory or via the optimization of the economic surplus in the framework of a competitive equilibrium. The theory proposed by Lucas and Prescott is now extremely classical and natural for economists. The theory of MFG broadens the domain of applications to situations in which, due to various economic frictions, the MFG equilibrium is not Pareto optimal and modelling via an equivalent central planner becomes impossible. 


\subsubsection{Two special cases}

\section{The demand law is of the form}

$$
D(X, p)=X(1-\epsilon p)_{+} .
$$

In this case, (11) becomes

$$
\begin{aligned}
0= & -r u+k 1_{\{k R \leq X\}}\left(\frac{1-\frac{k R}{X}}{\epsilon}-c-u\right)_{+}-\min \left((1-\epsilon(u+c))_{+}, \frac{k R}{X}\right) X \partial_{R} u \\
& +\partial_{R}\left(R \max _{\alpha}(u \phi(\alpha)-\alpha)\right)+b X \partial_{X} u+\frac{1}{2} \sigma^{2} X^{2} \partial_{X X} u,
\end{aligned}
$$

and the reduced equation(13) becomes

$$
\begin{aligned}
0= & -r v+\frac{k 1_{\{k y \leq 1\}}}{\epsilon}(1-\epsilon(v+c)-k y)_{+}-k y v^{\prime}+\left((1-\epsilon(v+c))_{+}-k y\right)_{-} v^{\prime} \\
& +\frac{d}{d y}\left(y \max _{\alpha}(v \phi(\alpha)-\alpha)\right)+\left(\sigma^{2}-b\right) y v^{\prime}+\frac{\sigma^{2} y^{2}}{2} v^{\prime \prime} .
\end{aligned}
$$

In (19), $H$ is given by (18) and $H_{1}$ is defined on $\mathbb{R}_{+} \times \mathbb{R}_{+}$by

$$
\begin{aligned}
H_{1}(y, v) & =-1_{\{1-\epsilon(v+c)<k y\}} \frac{(1-\epsilon(v+c))^{2}}{2 \epsilon}+1_{\{1-\epsilon(v+c) \geq k y\}} \frac{k y}{\epsilon}\left(\epsilon(v+c)-1+\frac{k y}{2}\right) \\
& =\frac{k y}{\epsilon}\left(\epsilon(v+c)-1+\frac{k y}{2}\right)-\frac{(\epsilon(v+c)-1+k y)_{+}^{2}}{2 \epsilon},
\end{aligned}
$$

which is obtained by choosing $M=\frac{1}{\epsilon}$ in (15). Then (19) becomes

$$
(r-b) V+(b+k) y V^{\prime}+H_{2}\left(y, V^{\prime}\right)+\frac{k y}{\epsilon}\left(\epsilon c-1+\frac{k y}{2}\right)-\frac{\left(\epsilon\left(V^{\prime}+c\right)-1+k y\right)_{+}^{2}}{2 \epsilon}-\frac{\sigma^{2} y^{2}}{2} V^{\prime \prime}=0 .
$$

If $\phi(\alpha)=C \sqrt{\alpha}$, then $H_{2}(v)=-y \frac{C^{2} v^{2}}{4}$ and (20) becomes

$$
\begin{aligned}
0= & (r-b) V+(b+k) y V^{\prime}-\frac{y}{4} C^{2}\left(V^{\prime}\right)^{2}-\frac{\sigma^{2} y^{2}}{2} V^{\prime \prime} \\
& +\frac{k y}{\epsilon}\left(\epsilon c-1+\frac{k y}{2}\right)-\frac{\left(\epsilon\left(V^{\prime}+c\right)-1+k y\right)_{+}^{2}}{2 \epsilon}-\frac{\sigma^{2} y^{2}}{2} V^{\prime \prime}=0 .
\end{aligned}
$$

The demand law is of the form

$$
D(X, p)=X p^{-s}
$$

In this case, (13) becomes

$$
\begin{aligned}
0= & -r v+k 1_{\left\{v+c \leq(k y)^{-1 / s\}}\right.}\left((k y)^{-1 / s}-c-v-y v^{\prime}\right)-1_{\left\{v+c>(k y)^{-1 / s}\right\}}(v+c)^{-s} v^{\prime} \\
& +\frac{d}{d y}\left(y \max _{\alpha}(v \phi(\alpha)-\alpha)\right)+\left(\sigma^{2}-b\right) y v^{\prime}+\frac{\sigma^{2} y^{2}}{2} v^{\prime \prime} .
\end{aligned}
$$

and the Hamiltonian $H_{1}$ takes the form:

$$
H_{1}(y, v)=k 1_{\left\{v+c \leq(k y)^{-1 / s}\right\}}\left(y(v+c)-\frac{s}{s-1} k^{-1 / s} y^{1-1 / s}\right)+\frac{1}{1-s} 1_{\left\{v+c>(k y)^{-1 / s}\right\}}(c+v)^{1-s} .
$$


If $\phi(\alpha)=C \sqrt{\alpha}$, then the Hamilton Jacobi equation is:

$$
\begin{aligned}
0= & (r-b) V+b y V^{\prime}-\frac{y}{4} C^{2}\left(V^{\prime}\right)^{2}-\frac{\sigma^{2} y^{2}}{2} V^{\prime \prime} \\
& +k 1_{\left\{V^{\prime}+c \leq(k y)^{-1 / s}\right\}}\left(y\left(V^{\prime}+c\right)-\frac{s}{s-1} k^{-1 / s} y^{1-1 / s}\right)+\frac{1}{1-s} 1_{\left\{V^{\prime}+c>(k y)^{-1 / s}\right\}}\left(c+V^{\prime}\right)^{1-s} .
\end{aligned}
$$

\subsection{A model of an open industry with a single technology}

Here, investment into prospection is not reserved to the existing units. The efficiency of the investment is assumed to have the same law has above: an investment rate of $\alpha$ results in increasing the reserves at a rate $\phi(\alpha)$. The global increase of the reserves if all units invest $\alpha$ (the global investment is then $\alpha R$ ) during $d t$ is $R \phi(\alpha) d t$. Globally, since there are no restriction to investment, the investment rate will be such that the marginal value created by a unitary investment is 1 , i.e.

$$
u \phi^{\prime}\left(\alpha^{*}\right)=1,
$$

where $u$ is the value of a production unit. If we assume that $\phi(\alpha)=C \sqrt{\alpha},(25)$ has a unique solution: $\alpha^{*}=C^{2} u^{2} / 4$. The evolution of the reserve is given by (10) with the same value of $\alpha^{*}$. All the other aspects of the model are described in $\S 2.1$. The value $u$ is obtained by optimizing on the control $\beta$ :

$$
u(R, X)=(1-r d t) \max _{0 \leq \beta \leq k} \mathbb{E}(\beta g(R, X, u) d t+(1-\beta d t) u(R+d R, X+d X))
$$

A first order expansion yields

$$
-r u(R, X)+k(g(R, X, u)-u(R, X))+\partial_{R} u \frac{d R}{d t}+\left(b X \partial_{X} u+\frac{1}{2} \sigma^{2} X^{2} \partial_{X X} u\right)=0
$$

where the optimal $\beta$ has been given by $\beta^{*}=k 1_{\{g(R, X, u)-u>0\}}$. We obtain the partial differential equation

$$
-r u+k(g(\cdot, u)-u)-Q^{*}(\cdot, u) \partial_{R} u+\phi\left(\alpha^{*}\right) \partial_{R} u+b X \partial_{X} u+\frac{1}{2} \sigma^{2} X^{2} \partial_{X X} u=0 .
$$

Looking for a nonnegative solution of (11) of the form $u(R, X)=v(y)$, we obtain the second order differential equation:

$$
\begin{aligned}
0= & -r v+k 1_{\left\{\tilde{D}^{-1}(k y) \geq v+c\right\}}\left(\tilde{D}^{-1}(k y)-c-v-y v^{\prime}\right)-1_{\{\tilde{D}(v+c)<k y\}} \tilde{D}(v+c) v^{\prime} \\
& +y \phi\left(\alpha^{*}\right) v^{\prime}+\left(\sigma^{2}-b\right) y v^{\prime}+\frac{\sigma^{2} y^{2}}{2} v^{\prime \prime},
\end{aligned}
$$

and $\phi\left(\alpha^{*}\right)=C^{2} v / 2$ if $\phi(\alpha)=C \sqrt{\alpha}$.

In this case, there is no related Hamilton-Jacobi equation.

\section{Mathematical analysis of Hamilton-Jacobi equations related to $(24)$}

The typical equation that we shall study is a simplified version of (24), namely

$$
v+H\left(x, \frac{d v}{d x}\right)=x^{-\alpha} \text { in }[0,+\infty),
$$

where $\alpha$ is a nonnegative number. 
Remark 3.1. Note that $v$ plays the role of $-V$ in (24). Compared to (24), we dropped the second order term and isolated the singularity of the data at $x=0$. We will tackle (24) in the case when $\sigma=b=0$ in $\S 3.3$ below.

Here, the Hamiltonian $H$ is a continuous real valued function on $[0,+\infty) \times \mathbb{R}$, and satisfies the following assumptions:

$\left(\mathbf{H}_{\mathbf{0}}\right) x \mapsto H(x, 0)$ is a bounded continuous function on $[0,+\infty)$ such that $H(0,0)=0$.

For some $m \geq 1$,

$\left(\mathbf{H}_{1}\right)$ there exist two positive constants $\nu \leq \mu$, a nonnegative function $C_{1} \in \mathrm{BC}([0,+\infty))$ with $C_{1}(0)=0$, a nonnegative function $C_{2} \in \mathrm{C}([0,+\infty))$ with $C_{2}(0)=0$ and $C_{2}(x) / x$ bounded in $(0,+\infty)$ such that $\forall x \in[0,+\infty), \forall p \in \mathbb{R}$,

$$
H(x, 0)+\nu x|p|^{m}-C_{2}(x) \leq H(x, p) \leq H(x, 0)+\mu x|p|^{m}+C_{1}(x)
$$

$\left(\mathbf{H}_{2}\right)$ there exists a modulus of continuity $\omega$ such that

$$
|H(x, p)-H(y, p)| \leq \omega\left(|x-y|\left(1+|p|^{m}\right)\right), \quad \forall x, y \in[0,+\infty), \forall p \in \mathbb{R}
$$

If $m>1$, there exists a modulus of continuity $\omega$ such that $\forall x, y \in(0,+\infty), \forall p \in \mathbb{R}$,

$$
\left|H\left(x, p x^{-\frac{1}{m}}\right)-H\left(y, p y^{-\frac{1}{m}}\right)\right| \leq \omega\left(\left|x^{\frac{m-1}{m}}-y^{\frac{m-1}{m}}\right|(1+|p|)\right)
$$

$\left(\mathbf{H}_{3}\right)$ For any $R>0$, there exists a modulus of continuity $\omega_{R}$ such that $\forall x \in[0,+\infty)$, for any $p, q \in \mathbb{R}$, if $-R \leq p \leq R$ and $-R \leq q \leq R$, then

$$
|H(x, p)-H(x, q)| \leq \omega_{R}(x|p-q|)
$$

$\left(\mathbf{H}_{4}\right)$ if $m>1$, there exist $\delta>0$ and two continuous Hamiltonians $H_{1}$ and $H_{2}$ such that $H(x, p)=H_{1}(x, p)+H_{2}(x, p)$ in $[0, \delta] \times \mathbb{R}, x \mapsto H_{1}(x, p)$ is continuous in $[0, \delta]$ uniformly with respect to $p \in \mathbb{R}, x \mapsto H_{2}(x, p)$ is nondecreasing in $[0, \delta]$ for any $p \in \mathbb{R}$

Remark 3.2. It can be checked that all the results stated and proved in $\S 3.1$ and 3.2 below hold if (29) is replaced by

$H(x, 0)+\nu x|p|^{m}-C_{2}(x) \leq H(x, p) \leq H(x, 0)+\mu x\left(|p|^{m}+|p|\right)+C_{1}(x), \quad \forall x \in[0,+\infty), \forall p \in \mathbb{R}$

with the same conditions as above on $C_{1}$ and $C_{2}$.

An issue is to understand what definition of viscosity solutions should be chosen for (28), in particular what condition should be imposed at $x=0$.

\subsection{Bounded right hand sides}

We first study

$$
v+H\left(x, \frac{d v}{d x}\right)=f(x) \quad \text { in }[0,+\infty)
$$

for $f \in \mathrm{BC}([0,+\infty))$. 


\subsubsection{The case when $m=1$}

In this paragraph, we suppose that Assumptions $\left(\mathbf{H}_{\mathbf{0}}\right)-\left(\mathbf{H}_{\mathbf{3}}\right)$ hold with $m=1$. The following comparison result for (34) can be proved with the same techniques as in Lions [13], BardiCapuzzo Dolcetta [3]:

Theorem 3.1. Consider two functions $u, w \in \operatorname{BUC}([0,+\infty))$. Under Assumptions $\left(\mathbf{H}_{\mathbf{0}}\right)-\left(\mathbf{H}_{\mathbf{3}}\right)$ with $m=1$, if $w$ is a supersolution of (34) and $u$ is a sub solution of (34) in $[0, \infty)$, then $u \leq w$.

Proof. We double the variables in $[0, \infty]$ and use a similar penalty function as in the proof of [3], II, Theorem 3.5, namely $\frac{1}{\epsilon}|x-y|^{2}+\beta(g(x)+g(y))$, where $g(x)=\frac{1}{2} \ln \left(1+(x-a)_{+}^{2}\right)$ for some fixed value of $a>0$ and $0<\beta<1$. The parameter $\beta$ will be chosen later. We study the maximum points of $\psi_{\epsilon}(x, y)=u(x)-w(y)-\frac{1}{\epsilon}|x-y|^{2}-\beta(g(x)+g(y))$.

Assume by contradiction that $M=\sup (u-w)>0$. Then there exists $x_{0}>0$ such that $b=u\left(x_{0}\right)-w\left(x_{0}\right)>0$. We can always choose $\bar{\beta}<1$ small enough such that for all $0<$ $\beta \leq \bar{\beta}, \beta g\left(x_{0}\right)<b / 4$. Let $\left(x_{\epsilon}, y_{\epsilon}\right)$ be such that $\psi_{\epsilon}\left(x_{\epsilon}, y_{\epsilon}\right)=\max _{x, y} \psi_{\epsilon}(x, y)>b / 2$. Clearly, $\frac{1}{\epsilon}\left|x_{\epsilon}-y_{\epsilon}\right|^{2}+\beta\left(g\left(x_{\epsilon}\right)+g\left(y_{\epsilon}\right)\right)$ is bounded uniformly with respect to $\epsilon$ and $\beta \in(0, \bar{\beta}]$. Therefore, as $\epsilon$ tend to 0 , we may assume that both $x_{\epsilon}$ and $y_{\epsilon}$ converge to some point $\bar{x} \in[0,+\infty)$. Moreover, $\lim _{\epsilon \rightarrow 0} \frac{1}{\epsilon}\left|x_{\epsilon}-y_{\epsilon}\right|^{2}=0$ since $\psi_{\epsilon}\left(x_{\epsilon}, x_{\epsilon}\right)+\psi_{\epsilon}\left(y_{\epsilon}, y_{\epsilon}\right) \leq 2 \psi_{\epsilon}\left(x_{\epsilon}, y_{\epsilon}\right)$.

Let us first focus on the case when $\bar{x} \geq a$. For $\epsilon$ small enough, we may assume that $x_{\epsilon}>a / 2$ and that $y_{\epsilon}>a / 2$. We use the notations $q_{1}=\frac{2}{\epsilon}\left(x_{\epsilon}-y_{\epsilon}\right)+\beta g^{\prime}\left(x_{\epsilon}\right)$ and $q_{2}=\frac{2}{\epsilon}\left(x_{\epsilon}-y_{\epsilon}\right)-\beta g^{\prime}\left(y_{\epsilon}\right)$. Since $u$ is a viscosity sub solution, $\left|q_{1}\right| \leq C$, where $C$ is the Lipschitz constant of $u$ in $[a / 2,+\infty)$ which is independent of $\epsilon$ and $\beta$. This implies that $2 \frac{\left|x_{\epsilon}-y_{\epsilon}\right|}{\epsilon} \leq C+1$ since $\left\|g^{\prime}\right\|_{\infty} \leq 1$. Then $\left|q_{2}\right| \leq C+2$. Set $Q=C+2$.

The viscosity inequalities are $u\left(x_{\epsilon}\right)+H\left(x_{\epsilon}, q_{1}\right) \leq f\left(x_{\epsilon}\right)$ and $w\left(y_{\epsilon}\right)+H\left(y_{\epsilon}, q_{2}\right) \geq f\left(y_{\epsilon}\right)$. From Assumption $\left(\mathbf{H}_{\mathbf{3}}\right)$,

$$
\left|H\left(x_{\epsilon}, q_{1}\right)-H\left(x_{\epsilon}, q_{2}\right)\right| \leq \omega_{Q}\left(\beta x_{\epsilon}\left(\left|g^{\prime}\left(x_{\epsilon}\right)\right|+\left|g^{\prime}\left(y_{\epsilon}\right)\right|\right)\right) .
$$

Note that $x_{\epsilon}\left|g^{\prime}\left(x_{\epsilon}\right)\right| \leq 1+a$ and that $x_{\epsilon}\left|g^{\prime}\left(y_{\epsilon}\right)\right| \leq 1+a+\left|x_{\epsilon}-y_{\epsilon}\right| \leq 2+a$ if $\epsilon$ is small enough. Hence, $\beta x_{\epsilon}\left(\left|g^{\prime}\left(x_{\epsilon}\right)\right|+\left|g^{\prime}\left(y_{\epsilon}\right)\right| \leq \beta(3+2 a)\right.$. From Assumption $\left(\mathbf{H}_{\mathbf{2}}\right)$,

$$
\left|H\left(x_{\epsilon}, q_{2}\right)-H\left(y_{\epsilon}, q_{2}\right)\right| \leq \omega\left(\left|x_{\epsilon}-y_{\epsilon}\right|(1+Q)\right) .
$$

Subtracting the two viscosity inequalities, using (35) and (36) and letting $\epsilon$ tend to 0, using also the continuity of $f$, yields that $b / 2 \leq u(\bar{x})-w(\bar{x}) \leq \omega_{Q}(\beta(3+2 a))$. For $\beta$ small enough, this is a contradiction.

Now, we consider the case when $\bar{x}<a$. Then $u(\bar{x})-w(\bar{x})=M$. For $\epsilon$ small enough, we may assume that $x_{\epsilon}<a$ and $y_{\epsilon}<a$. For $q_{\epsilon}=\frac{x_{\epsilon}-y_{\epsilon}}{\epsilon}$, the viscosity inequalities are $u\left(x_{\epsilon}\right)+$ $H\left(x_{\epsilon}, q_{\epsilon}\right) \leq f\left(x_{\epsilon}\right)$ and $w\left(y_{\epsilon}\right)+H\left(y_{\epsilon}, q_{\epsilon}\right) \geq f\left(y_{\epsilon}\right)$. Subtracting and using Assumption $\left(\mathbf{H}_{\mathbf{2}}\right)$ yields $u\left(x_{\epsilon}\right)-w\left(y_{\epsilon}\right) \leq f\left(x_{\epsilon}\right)-f\left(y_{\epsilon}\right)+\omega\left(\left|x_{\epsilon}-y_{\epsilon}\right|\left(1+\left|q_{\epsilon}\right|\right)\right)$. Since $\lim _{\epsilon \rightarrow 0}\left|x_{\epsilon}-y_{\epsilon}\right|\left|q_{\epsilon}\right|=0$, we obtain that $M \leq 0$ by passing to the limit.

An existence result can also be obtained. We skip the proof for brevity.

Theorem 3.2. Under Assumptions $\left(\mathbf{H}_{\mathbf{0}}\right)-\left(\mathbf{H}_{\mathbf{3}}\right)$ with $m=1$, there exists a unique viscosity solution $v \in \mathrm{BUC}([0,+\infty))$ of (34).

Value at $x=0 \quad$ Recall that from the assumptions, $H(x, p) \leq H(x, 0)+\mu x|p|+C_{1}(x)$ and that $H(\cdot, 0)$ and $C_{1}$ are bounded and continuous functions vanishing at 0 . Consider the following 
optimal control problem:

$u(x)=\inf \left\{\begin{array}{l|l}\int_{0}^{+\infty} e^{-s}\left(f\left(z_{x}(s ; \alpha)\right)-H\left(z_{x}(s ; \alpha), 0\right)-C_{1}\left(z_{x}(s ; \alpha)\right)\right) d s ; & \begin{array}{l}\dot{z}_{x}(s ; \alpha)=\alpha(s) z_{x}(s ; \alpha) \\ z_{x}(0 ; \alpha)=x \\ \alpha(s)= \pm \mu\end{array}\end{array}\right\}$.

Clearly, $u(0)=f(0)$ and $u$ is a viscosity sub solution of (34). On the other hand, there exists a uniformly continuous function $\tilde{w}$ on $[0,+\infty)$ such that $\tilde{w}(0)=f(0), \tilde{w}(x) \geq-H(x, p)+f(x)$ for any $x \in[0,+\infty)$ and $p \in \mathbb{R}$. Consider $M=\sup _{x}(f(x)-H(x, 0)) \geq f(0)$ and call $w=\min (M, \tilde{w})$. The function $w$ is a viscosity supersolution of $(34)$ in $[0,+\infty)$. Then, from Theorem 3.1 , the bounded viscosity solution $v$ of (34) is such that $u \leq v \leq w$ : therefore $v(0)=f(0)$.

\subsubsection{The case when $m>1$}

Proposition 3.1. Under Assumptions $\left(\mathbf{H}_{\mathbf{0}}\right)-\left(\mathbf{H}_{\mathbf{3}}\right)$ with $m>1$, there exists a viscosity solution $v \in \mathrm{BUC}([0,+\infty))$ of (34). It satisfies $v(0) \leq f(0)$.

Proof. For a positive number $M$, we approximate (34) by

$$
v+H_{M}\left(x, \frac{d v}{d x}\right)=f(x), \quad \text { in }[0,+\infty)
$$

where $H_{M}(x, p)=\min (\nu M x|p|+H(x, 0), H(x, p))$. The function $H_{M}$ satisfies assumptions $\left(\mathbf{H}_{\mathbf{0}}\right)$ and $\left(\mathbf{H}_{\mathbf{1}}\right)$ with $m=1$. Indeed,

$\nu x M|p|+H(x, 0)-C_{2}(x)-\nu x M\left(\frac{M}{m}\right)^{\frac{1}{m-1}} \leq H_{M}(x, p) \leq \nu M x|p|+H(x, 0), \quad \forall x \geq 0, \forall p \in \mathbb{R}$.

Take $x, y \in[0,+\infty)$ and $p \in \mathbb{R}$ : we can make out three cases:

1. If $H(x, p)<\nu M x|p|+H(x, 0)$ and $H(y, p)<\nu M y|p|+H(y, 0)$, then $x y>0$ and $\nu|p|^{m} \leq$ $\nu M|p|+C_{2}(x) / x$. Note that $\tilde{C}(x)=C_{2}(x) / x$ is bounded, (recall that $C_{2}(0)=0$ and that $C_{2}(x) / x$ is bounded). Hence, $\nu|p|^{m} \leq \nu M|p|+\|\tilde{C}\|_{\infty}$ and $\left|H_{M}(x, p)-H_{M}(y, p)\right| \leq$ $\omega\left(|x-y|\left(1+M|p|+\|\tilde{C}\|_{\infty} / \nu\right)\right)$.

2. If $H_{M}(x, p)=\nu M x|p|+H(x, 0)$ and $H_{M}(y, p)=\nu M y|p|+H(y, 0)$, then $\mid H_{M}(x, p)-$ $H_{M}(y, p)|\leq \nu M| x-y|| p \mid+\omega(|x-y|)$.

3. If $H_{M}(x, p)=\nu M x|p|+H(x, 0)$ and $H(y, p)<\nu M y|p|+H(y, 0)$, then there must exist some $z$ between $x$ and $y$ such that $H(z, p)=\nu M z|p|+H(z, 0)$. In that case,

$$
\begin{aligned}
\left|H_{M}(x, p)-H_{M}(y, p)\right| & \leq|H(y, p)-H(z, p)|+\nu M|x-z||p|+\omega(|x-z|) \\
& \leq \omega\left(|z-y|\left(1+M|p|+\|\tilde{C}\|_{\infty} / \nu\right)\right)+\nu M|x-z||p|+\omega(|x-z|) \\
& \leq \omega\left(|x-y|\left(1+M|p|+\|\tilde{C}\|_{\infty} / \nu\right)\right)+\nu M|x-y||p|+\omega(|x-y|) .
\end{aligned}
$$

To summarize, $H_{M}$ satisfies Assumption $\left(\mathbf{H}_{\mathbf{2}}\right)$ with $m=1$.

It is also clear that if $|p| \leq R$ and $|q| \leq R$, then for any $x \geq 0,\left|H_{M}(x, p)-H_{M}(x, q)\right| \leq$ $\nu M x|p-q|+\omega_{R}(x|p-q|)$ so Assumption $\left(\mathbf{H}_{\mathbf{3}}\right)$ is satisfied by $H_{M}$.

From Theorems 3.1 and 3.2 , we know that there is a unique viscosity solution $v_{M} \in$ $\operatorname{BUC}([0,+\infty))$ of $(37)$. Moreover $v_{M}(0)=f(0)$. 
The comparison principle ensures that the family of functions $\left(v_{M}\right)_{M>0}$ is nonincreasing with respect to $M: v_{N} \leq v_{M}$ if $N \geq M$, and that $v_{N} \geq \inf _{x}(f(x)-H(x, 0))$.

It can be deduced from the latter observation that for all $y>0, v_{M}$ is a viscosity sub solution of $\min \left(\nu\left|\frac{d v_{M}}{d x}\right|^{m}, \nu M\left|\frac{d v_{M}}{d x}\right|\right) \leq c_{y}$ in $[y,+\infty)$, where $c_{y}$ depends on $y$ but not on $M$. If $M>\left(c_{y} / \nu\right)^{1-1 / m}$, then $v_{M}$ is a viscosity sub solution of $\left|v_{M}^{\prime}\right| \leq\left(c_{y} / \nu\right)^{1 / m}$ in $[y,+\infty)$ : therefore $v_{M}$ is continuous in $[y,+\infty)$ uniformly with respect to $M$.

The sequence $v_{M}$ converges in a monotone way to a function $v$ defined on $(0,+\infty)$, and the convergence is uniform in the compact subsets of $(0,+\infty)$. Therefore $v \in \mathrm{BC}(0, \infty)$ and $v \geq \inf _{x}(f(x)-H(x, 0))$. By standard stability results, $v$ is a viscosity solution of (37) in $(0,+\infty)$.

For $c=\sup _{x} f(x)-\inf _{x}(f(x)-H(x, 0)), v$ is a viscosity sub solution of $x\left|v^{\prime}\right|^{m} \leq \frac{c}{\nu}$ in $(0,+\infty)$, which implies that

$$
|v(x)-v(y)| \leq\left(\frac{c}{\nu}\right)^{\frac{1}{m}} \frac{m}{m-1}\left|x^{1-\frac{1}{m}}-y^{1-\frac{1}{m}}\right|, \quad \forall 0<x, y .
$$

This shows that $v$ is uniformly continuous on $(0, z]$ for all $z>0$ and can be extended to a continuous function (still named $v$ ) defined in $[0,+\infty)$. Moreover, since $v_{M}(0)=f(0)$ and $v_{M}$ converges in a nonincreasing manner in $(0,+\infty), v(0) \leq f(0)$. Hence, $v$ is a sub solution of $(34)$ in $[0,+\infty)$. We notice that $v(0)=\liminf _{x \rightarrow 0^{+}, M \rightarrow+\infty} v_{M}(x)$.

We now claim that one among the following two assertions is true

1. $v(0)=f(0)$

2. $v(0)<f(0)$ and for any function $\phi \in C^{1}([0,+\infty)), v-\phi$ does not have a local minimum at 0 .

As a consequence, $v$ is a supersolution of $(34)$ in $[0,+\infty)$.

The claim is proved by contradiction: assume that $v(0)<f(0)$ and that $v-\phi$ has a local minimum at 0 . Replacing possibly $\phi$ by $\phi-x^{2}$, we may also suppose that $v-\phi$ has a strict local minimum at 0 . Since $v(0)=\liminf _{x \rightarrow 0^{+}, M \rightarrow+\infty} v_{M}(x)$, classical arguments show that there exists a sequence $\left(M_{n}\right)_{n>0}$ such that $\lim _{n \rightarrow \infty} M_{n}=+\infty$, a sequence of positive numbers $\left(x_{n}\right)_{n>0}$ such that $v_{M_{n}}-\phi$ has a local minimum at $x_{n}, \lim _{n \rightarrow \infty} x_{n}=0$ and $\lim _{n \rightarrow \infty} v_{M_{n}}\left(x_{n}\right)=$ $v(0)$. A key point is to observe that there cannot exist a subsequence (not relabeled) such that $x_{n}=0$, because in this case, $v_{M_{n}}(0)=f(0)$ would imply $v(0)=f(0)$. Therefore, $v_{M_{n}}\left(x_{n}\right)+$ $H_{M_{n}}\left(x_{n}, \frac{d \phi}{d x}\left(x_{n}\right)\right) \geq f\left(x_{n}\right)$, and since $\phi \in C^{1}([0,+\infty))$, this yields for $n$ large enough: $v_{M_{n}}\left(x_{n}\right)+$ $H\left(x_{n}, \frac{d \phi}{d x}\left(x_{n}\right)\right) \geq f\left(x_{n}\right)$. Letting $n \rightarrow \infty$ yields that $\lim _{n \rightarrow \infty}\left|\frac{d \phi}{d x}\left(x_{n}\right)\right|=+\infty$, i.e. the desired contradiction.

Theorem 3.3. Under Assumptions $\left(\mathbf{H}_{\mathbf{0}}\right)-\left(\mathbf{H}_{4}\right)$ with $m>1$, if $w \in \operatorname{BUC}([0,+\infty))$ is a supersolution of (34) and $u \in \operatorname{BUC}([0,+\infty))$ is a sub solution of (34) in $[0,+\infty)$, then $u \leq w$.

Proof. In the spirit of the comparison principles proved by Soner [15, 16], Capuzzo DolcettaLions [4], see also [3], for problems with state constraint boundary conditions, we choose a monotone function $T \in C^{1}([0,+\infty))$ such that $T(x)=1$ in $[0, h]$, and $T(x)=0$ in $[2 h,+\infty)$, for some $h<\delta$.

Assume first that the supremum $M$ of $u-w$ is not achieved at $x=0$. In this case, the same proof as for Theorem 3.1 can be used, mainly because $H$ is uniformly coercive away from $x=0$ : it consists of studying the maximum points of the function $\psi_{\epsilon}(x, y)=u(x)-w(y)-\frac{1}{\epsilon}|x-y|^{2}-$ $\beta(g(x)+g(y))$, where $g(x)=\frac{1}{2} \ln \left(1+(x-\delta)_{+}^{2}\right)$. It yields that $M \leq 0$. 
Assume now that the maximum $M$ of $u-w$ is achieved at $x=0$ and consider the function $\psi_{\epsilon}(x, y)=u(x)-w(y)-\frac{1}{\epsilon}\left|x^{\frac{m-1}{m}}-(y+\sqrt{\epsilon} T(y))^{\frac{m-1}{m}}\right|^{2}-(g(x)+g(y))$. The supremum of $\psi_{\epsilon}$ in $[0,+\infty)^{2}$ is a maximum. Since $u(0)-w(0)=M$, we see that $\max \psi_{\epsilon}(x, y) \geq \psi_{\epsilon}(\sqrt{\epsilon}, 0) \geq$ $M-\omega_{u}(\sqrt{\epsilon})$, where $\omega_{u}$ is the modulus of continuity of $u$. Therefore, if $\left(x_{\epsilon}, y_{\epsilon}\right)$ a maximum point of $\psi_{\epsilon}$, then $x_{\epsilon}, y_{\epsilon}$ and $\frac{1}{\epsilon}\left|x^{\frac{m-1}{m}}-(y+\sqrt{\epsilon} T(y))^{\frac{m-1}{m}}\right|^{2}$ are bounded by some $R$ independent of $\epsilon$. Therefore, after the extraction of a subsequence, we can assume that both $x_{\epsilon}$ and $y_{\epsilon}$ converge to some point $\bar{x} \in[0,+\infty)$. Moreover,

$$
\psi_{\epsilon}\left(x_{\epsilon}, y_{\epsilon}\right) \leq M+\omega_{w}\left(\left|x_{\epsilon}-y_{\epsilon}\right|\right)-\frac{1}{\epsilon}\left|\left(y_{\epsilon}+\sqrt{\epsilon} T\left(y_{\epsilon}\right)\right)^{\frac{m-1}{m}}-x_{\epsilon}^{\frac{m-1}{m}}\right|^{2}-\left(g\left(x_{\epsilon}\right)+g\left(y_{\epsilon}\right)\right),
$$

where $\omega_{w}$ is the modulus of continuity of $w$. Combining the latter two observations,

$$
\frac{1}{\epsilon}\left|\left(y_{\epsilon}+\sqrt{\epsilon} T\left(y_{\epsilon}\right)\right)^{\frac{m-1}{m}}-x_{\epsilon}^{\frac{m-1}{m}}\right|^{2}+\left(g\left(x_{\epsilon}\right)+g\left(y_{\epsilon}\right)\right) \leq \omega_{w}\left(\left|x_{\epsilon}-y_{\epsilon}\right|\right)+\omega_{u}(\sqrt{\epsilon}),
$$

which implies that

$$
\left|\left(y_{\epsilon}+\sqrt{\epsilon} T\left(y_{\epsilon}\right)\right)^{\frac{m-1}{m}}-x_{\epsilon}^{\frac{m-1}{m}}\right| \leq \epsilon^{\frac{1}{2}} \eta(\epsilon) \quad \text { with } \lim _{\epsilon \rightarrow 0} \eta(\epsilon)=0
$$

and that

$$
g\left(x_{\epsilon}\right)+g\left(y_{\epsilon}\right) \leq \eta(\epsilon) .
$$

If for a subsequence, $y_{\epsilon} \in(h, R]$, then we may assume that $x_{\epsilon} \in(h / 2, R]$. Of course, $\bar{x} \in[h, R]$. We then use the Lipschitz continuity of $u$ in $[h / 2,+\infty)$ and obtain that

$$
\left|\frac{2}{\epsilon} \frac{m-1}{m}\left(x_{\epsilon}^{\frac{m-1}{m}}-\left(y_{\epsilon}+\sqrt{\epsilon} T\left(y_{\epsilon}\right)\right)^{\frac{m-1}{m}}\right) x_{\epsilon}^{-\frac{1}{m}}+g^{\prime}\left(x_{\epsilon}\right)\right| \leq L_{u},
$$

where $L_{u}$ is the Lipschitz constant of $u$ in $[h / 2,+\infty)$. Next, setting

$$
\begin{aligned}
q & =\frac{2}{\epsilon} \frac{m-1}{m}\left(x_{\epsilon}^{\frac{m-1}{m}}-\left(y_{\epsilon}+\sqrt{\epsilon} T\left(y_{\epsilon}\right)\right)^{\frac{m-1}{m}}\right)-g^{\prime}\left(y_{\epsilon}\right) y_{\epsilon}^{\frac{1}{m}}, \\
q_{1} & =\frac{2}{\epsilon} \frac{m-1}{m}\left(x_{\epsilon}^{\frac{m-1}{m}}-\left(y_{\epsilon}+\sqrt{\epsilon} T\left(y_{\epsilon}\right)\right)^{\frac{m-1}{m}}\right) x_{\epsilon}^{-\frac{1}{m}}+g^{\prime}\left(x_{\epsilon}\right), \\
q_{2} & =\frac{2}{\epsilon} \frac{m-1}{m}\left(x_{\epsilon}^{\frac{m-1}{m}}-\left(y_{\epsilon}+\sqrt{\epsilon} T\left(y_{\epsilon}\right)\right)^{\frac{m-1}{m}}\right)\left(y_{\epsilon}+\sqrt{\epsilon} T\left(y_{\epsilon}\right)\right)^{-\frac{1}{m}}\left(1+\sqrt{\epsilon} T^{\prime}\left(y_{\epsilon}\right)\right)-g^{\prime}\left(y_{m}\right), \\
\tilde{q}_{1} & =\frac{2}{\epsilon} \frac{m-1}{m}\left(x_{\epsilon}^{\frac{m-1}{m}}-\left(y_{\epsilon}+\sqrt{\epsilon} T\left(y_{\epsilon}\right)\right)^{\frac{m-1}{m}}\right) x_{\epsilon}^{-\frac{1}{m}}-g^{\prime}\left(y_{\epsilon}\right) y_{\epsilon}^{\frac{1}{m}} x_{\epsilon}^{-\frac{1}{m}}, \\
\tilde{q}_{2} & =\frac{2}{\epsilon} \frac{m-1}{m}\left(x_{\epsilon}^{\frac{m-1}{m}}-\left(y_{\epsilon}+\sqrt{\epsilon} T\left(y_{\epsilon}\right)\right)^{\frac{m-1}{m}}\right) y_{\epsilon}^{-\frac{1}{m}}-g^{\prime}\left(y_{\epsilon}\right),
\end{aligned}
$$

the previous estimates show that there exist positive constant $Q$ and $C$ independent of $\epsilon$ (if $\epsilon$ is small enough) such that $\max \left(|q|,\left|q_{1}\right|,\left|q_{2}\right|,\left|\tilde{q}_{1}\right|,\left|\tilde{q}_{2}\right|\right) \leq Q$ and that $\left|q_{2}-\tilde{q}_{2}\right| \leq C \sqrt{\epsilon}$.

The viscosity inequalities are $u\left(x_{\epsilon}\right)+H\left(x_{\epsilon}, q_{1}\right) \leq f\left(x_{\epsilon}\right)$ and $w\left(y_{\epsilon}\right)+H\left(y_{\epsilon}, q_{2}\right) \geq f\left(y_{\epsilon}\right)$. Then,

$$
\begin{aligned}
\left|H\left(x_{\epsilon}, q_{1}\right)-H\left(x_{\epsilon}, \tilde{q}_{1}\right)\right| & \leq \omega_{Q}\left(\left|x_{\epsilon}\right|\left(g\left(x_{\epsilon}\right)+g\left(y_{\epsilon}\right) y_{\epsilon}^{\frac{1}{m}} x_{\epsilon}^{-\frac{1}{m}}\right)\right), \\
\left|H\left(y_{\epsilon}, q_{2}\right)-H\left(y_{\epsilon}, \tilde{q}_{2}\right)\right| & \leq \omega_{Q}\left(C\left|y_{\epsilon}\right| \sqrt{\epsilon}\right), \\
\left|H\left(x_{\epsilon}, \tilde{q}_{1}\right)-H\left(y_{\epsilon}, \tilde{q}_{2}\right)\right| & \leq \omega\left(\left|x_{\epsilon}^{\frac{m-1}{m}}-y_{\epsilon}^{\frac{m-1}{m}}\right|(1+|q|)\right) .
\end{aligned}
$$


Indeed (41) and (42) come from Assumption $\left(\mathbf{H}_{\mathbf{3}}\right)$ and (43) is a consequence of Assumption $\left(\mathbf{H}_{2}\right)$, namely (31). The right hand sides of (41)-(43) tend to 0 as $\epsilon \rightarrow 0$. Subtracting the viscosity inequalities, using (41)-(43) and (40), then passing to the limit yield that

$$
M=u(\bar{x})-w(\bar{x}) \leq 0 .
$$

There remains to discuss the case when for $\epsilon$ small enough, $y_{\epsilon} \in[0, h)$. In this case $T\left(y_{\epsilon}\right)=1$, $T^{\prime}\left(y_{\epsilon}\right)=0$ and $g\left(y_{\epsilon}\right)=g\left(x_{\epsilon}\right)=0$ for $\epsilon$ small enough. We make out two cases:

1. $y_{\epsilon}>0$ : we know that $x_{\epsilon}>0$ because $\left|\left(y_{\epsilon}+\sqrt{\epsilon}\right)^{\frac{m-1}{m}}-x_{\epsilon}^{\frac{m-1}{m}}\right| \leq \epsilon^{\frac{1}{2}} \eta(\epsilon)$ with $\lim _{\epsilon \rightarrow 0} \eta(\epsilon)=$ 0 ; defining $q_{\epsilon}=\frac{2}{\epsilon} \frac{m-1}{m}\left(x_{\epsilon}^{\frac{m-1}{m}}-\left(y_{\epsilon}+\sqrt{\epsilon}\right)^{\frac{m-1}{m}}\right)$ and subtracting the viscosity inequalities, we obtain

$$
u\left(x_{\epsilon}\right)-w\left(y_{\epsilon}\right)+H\left(x_{\epsilon}, q_{\epsilon} x_{\epsilon}^{-\frac{1}{m}}\right)-H\left(y_{\epsilon}, q_{\epsilon}\left(y_{\epsilon}+\sqrt{\epsilon}\right)^{-\frac{1}{m}}\right) \leq f\left(x_{\epsilon}\right)-f\left(y_{\epsilon}\right) .
$$

Then

$$
\begin{aligned}
& H\left(x_{\epsilon}, q_{\epsilon} x_{\epsilon}^{-\frac{1}{m}}\right)-H\left(y_{\epsilon}, q_{\epsilon}\left(y_{\epsilon}+\sqrt{\epsilon}\right)^{-\frac{1}{m}}\right) \\
\geq & -\left|H\left(x_{\epsilon}, q_{\epsilon} x_{\epsilon}^{-\frac{1}{m}}\right)-H\left(y_{\epsilon}+\sqrt{\epsilon}, q_{\epsilon}\left(y_{\epsilon}+\sqrt{\epsilon}\right)^{-\frac{1}{m}}\right)\right| \\
& -\left|H_{1}\left(y_{\epsilon}+\sqrt{\epsilon}, q_{\epsilon}\left(y_{\epsilon}+\sqrt{\epsilon}\right)^{-\frac{1}{m}}\right)-H_{1}\left(y_{\epsilon}, q_{\epsilon}\left(y_{\epsilon}+\sqrt{\epsilon}\right)^{-\frac{1}{m}}\right)\right| \\
& +H_{2}\left(y_{\epsilon}+\sqrt{\epsilon}, q_{\epsilon}\left(y_{\epsilon}+\sqrt{\epsilon}\right)^{-\frac{1}{m}}\right)-H_{2}\left(y_{\epsilon}, q_{\epsilon}\left(y_{\epsilon}+\sqrt{\epsilon}\right)^{-\frac{1}{m}}\right) .
\end{aligned}
$$

The first term in the right hand side of (45) tends to 0 from (31) and (39). The second term tends to 0 using the continuity of $H_{1}$, see Assumption $\left(\mathbf{H}_{\mathbf{4}}\right)$. The third term is nonnegative since $H_{2}$ is nondecreasing w.r.t $x$ by Assumption $\left(\mathbf{H}_{\mathbf{4}}\right)$.

Combining these observations, we deduce from (44) that $M \leq 0$ by letting $\epsilon$ tend to 0 .

2. If $y_{\epsilon}=0$, then $y \mapsto w(y)+\frac{1}{\epsilon}\left|x^{\frac{m-1}{m}}-(y+\sqrt{\epsilon})^{\frac{m-1}{m}}\right|^{2}$ has a minimum at 0 : this implies that $w(0) \geq f(0)$ and that $M \leq 0$ since $u(0) \leq f(0)$.

\subsection{Analysis of (28)}

We still make Assumptions $\left(\mathbf{H}_{\mathbf{0}}\right)-\left(\mathbf{H}_{\mathbf{4}}\right)$ with $m>1$ and suppose furthermore that $0<\alpha<m-1$.

Definition 3.1. We say that $v \in \mathrm{BUC}([0,+\infty))$ is a viscosity solution of (28) if it is a viscosity solution of (28) in $(0,+\infty)$ and if it is not possible to find a $C^{1}$ function $\phi$ such that $v-\phi$ has a local minimum at 0 .

Proposition 3.2. Under Assumptions $\left(\mathbf{H}_{\mathbf{0}}\right)-\left(\mathbf{H}_{\mathbf{4}}\right)$ with $m>1$, if $0<\alpha<m-1$, there exists a viscosity solution $v \in \mathrm{BUC}([0,+\infty))$ of (28).

Proof. There exist four constants $k_{1}, k_{2}>0, k_{3}=-\sup _{x} H(x, 0), \bar{h}: 0<\bar{h}<\delta$ (where $\delta$ is the constant appearing in the assumptions), such that $u_{h}(x)=\max \left(k_{1}-k_{2}(x+h)^{(m-1-\alpha) / m}, k_{3}\right)$ is a sub solution of $(28)$ in $(0,+\infty)$, for all $h, 0<h<\bar{h}$. Calling $R(h)=k_{1}+2 \mu\left(k_{2}(m-1-\right.$ $\alpha) / m)^{m} h^{-\alpha}$, it is always possible to decrease $\bar{h}$ in such a way that for any $0<h<\bar{h}, R(h)>0$ 
and $u_{h}$ is also a sub solution of $u_{h}+H\left(x, \frac{d u_{h}}{d x}\right) \leq \min \left(x^{-\alpha}, R(h)\right)$ in $[0,+\infty)$. From the previous paragraph, we know that there exists a unique viscosity solution $v_{h} \in \mathrm{BUC}([0,+\infty))$ of

$$
v_{h}+H\left(x, \frac{d v_{h}}{d x}\right)=\min \left(x^{-\alpha}, R(h)\right) \quad \text { in }[0,+\infty) .
$$

Comparison results imply that $v_{h} \geq k_{3}$ and that the family $\left(v_{h}\right)_{h}$ is nonincreasing with respect to $h$.

It is also possible to find a bounded supersolution $w$ of $(28)$ in $(0, \infty)$ of the form $w(x)=$ $K_{1}-K_{2} \min (x, 1)^{(m-1-\alpha) / m}$. Note that $w$ is also a supersolution of $(46)$ in $[0,+\infty)$.

Hence, $k_{3} \leq u_{h} \leq v_{h} \leq w$, and we see that $\left|v_{h}\right|$ is bounded uniformly in $h$; furthermore, $v_{h}$ is a viscosity sub solution of $\nu x\left|\frac{d v_{h}}{d x}\right|^{m} \leq x^{-\alpha}-k_{3}+C(x)$; this shows that for any $y>0$, the norms $\left\|v_{h}\right\|_{C^{(m-1-\alpha) / m}([0, y])}$ are bounded uniformly with respect to $h$.

From the monotonicity of the sequence $\left(v_{h}\right)_{h}$ and the uniform Hölder estimate, $v_{h}$ converges uniformly in the intervals $[0, y], y>0$ to some $\underline{v} \in \mathrm{BC}([0, \infty))$ such that $\underline{v} \in C^{(m-1-\alpha) / m}([0, y])$ for any $y>0$. The function $\underline{v}$ is a viscosity solution of $(28)$ in $(0,+\infty)$ and $\underline{v} \in \operatorname{BUC}([0, \infty))$. We also claim that it is not possible to find a $C^{1}$ function $\phi$ such that $\underline{v}-\phi$ has a local minimum at 0 . Indeed, in the opposite case, we could always assume that the minimum is strict by replacing $\phi$ by $\phi-x^{2}$, and by standard arguments, we could find a sequence $\left(h_{n}\right)_{n>0}$ such that $\lim _{n \rightarrow \infty} h_{n}=0$, a sequence of positive numbers $\left(x_{n}\right)_{n>0}$ such that $v_{h_{n}}-\phi$ has a local minimum at $x_{n}, \lim _{n \rightarrow \infty} x_{n}=0$ and $\lim _{n \rightarrow \infty} v_{h_{n}}\left(x_{n}\right)=\underline{v}(0)$. This would imply that $v_{h_{n}}\left(x_{n}\right)+H\left(x_{n}, \frac{d \phi}{d x}\left(x_{n}\right)\right) \geq \min \left(x_{n}^{-\alpha}, R\left(h_{n}\right)\right)$. This would yield that $\lim _{n \rightarrow \infty}\left|\frac{d \phi}{d x}\left(x_{n}\right)\right|=+\infty$, the desired contradiction. We have proved that $\underline{v}$ is a solution of (28).

Moreover, if $\tilde{v} \in \operatorname{BUC}([0,+\infty))$ is another viscosity solution of $(28)$, then $\tilde{v}$ is a supersolution of (46). Hence $\tilde{v} \geq v_{h}$, which shows that $\tilde{v} \geq \underline{v}: \underline{v}$ is the minimal solution of (28).

Proposition 3.3. Under Assumptions $\left(\mathbf{H}_{\mathbf{0}}\right)-\left(\mathbf{H}_{\mathbf{4}}\right)$ with $m>1$, if $w \in \mathrm{BUC}([0,+\infty))$ is a supersolution of (28) and $u \in \mathrm{BUC}([0,+\infty))$ is a sub solution of (28), then $u \leq w$.

Proof. The proof is identical to that of Theorem 3.3 until the discussion of the case when $y_{\epsilon} \in[0, h]$; at this point, the proof slightly differs as follows:

1. if $y_{\epsilon}>0$, then, subtracting the viscosity inequalities, we get

$$
u\left(x_{\epsilon}\right)-w\left(y_{\epsilon}\right)+H\left(x_{\epsilon}, q_{\epsilon} x_{\epsilon}^{-\frac{1}{m}}\right)-H\left(y_{\epsilon}, q_{\epsilon}\left(y_{\epsilon}+\sqrt{\epsilon}\right)^{-\frac{1}{m}}\right) \leq x_{\epsilon}^{-\alpha}-y_{\epsilon}^{-\alpha},
$$

which is the counterpart of (44), recalling that $q_{\epsilon}=\frac{2}{\epsilon} \frac{m-1}{m}\left(x_{\epsilon}^{\frac{m-1}{m}}-\left(y_{\epsilon}+\sqrt{\epsilon}\right)^{\frac{m-1}{m}}\right)$.

But we also know that

$$
\left|\left(y_{\epsilon}+\sqrt{\epsilon}\right)^{\frac{m-1}{m}}-x_{\epsilon}^{\frac{m-1}{m}}\right| \leq \epsilon^{\frac{1}{2}} \eta(\epsilon) \quad \text { with } \lim _{\epsilon \rightarrow 0} \eta(\epsilon)=0
$$

which implies that $x_{\epsilon}>y_{\epsilon}$ and that $x_{\epsilon}^{-\alpha} \leq y_{\epsilon}^{-\alpha}$ for $\epsilon$ small enough. From this, the fact that $M \leq 0$ follows as in the proof of Theorem 3.3.

2. The case $y_{\epsilon}=0$ is not possible since otherwise $y \mapsto w(y)+\frac{1}{\epsilon}\left|x^{\frac{m-1}{m}}-(y+\sqrt{\epsilon})^{\frac{m-1}{m}}\right|^{2}$ would have a minimum at 0 . 
Remark 3.3. Under Assumptions $\left(\mathbf{H}_{\mathbf{0}}\right)-\left(\mathbf{H}_{\mathbf{4}}\right)$ with $m>1$, if $\alpha \geq m-1$, it would be possible to prove that there exists a unique viscosity solution $v \in C(0,+\infty)$ of (28) which blows up at 0 (like $x^{(m-1-\alpha) / m}$ if $\alpha>m-1$ and logarithmically if $\alpha=m-1$ ). For brevity, we do no study this case in details; the proof of the existence of a minimal viscosity solution would be rather similar to the proof of Proposition 3.2. For the proof of uniqueness, a different strategy close to the one introduced in [9] would be needed.

\subsection{Analysis of (24) in the case when $\sigma=b=0$}

We aim at proving existence and uniqueness of a nondecreasing solution of (24), focusing on the case $\sigma=b=0$ for simplicity. After the change of variables $v=-V, x=y$, the equation takes the form

$$
\alpha v+H_{1}\left(x, v^{\prime}\right)+H_{2}\left(x, v^{\prime}\right)=\frac{s}{1-s} k^{1-1 / s} x^{1-1 / s},
$$

where $\alpha>0,1 / 2<s<1, H_{1}(x, p)=\gamma x p^{2}, \gamma>0$, and

$$
H_{2}(x, p)=1_{\left\{c-p>(k x)^{-1 / s}\right\}}\left(-\frac{1}{1-s}(c-p)^{1-s}+\frac{s}{1-s} k^{1-1 / s} x^{1-1 / s}\right)+1_{\left\{c-p \leq(k x)^{-1 / s}\right\}} k x(p-c),
$$

with $k>0, c>0$.

We can see formally that taking $\sigma>0$ would not change the singularity at $x=0$ : indeed, the singular behaviour found in $\S 3.2$ is $v^{\prime}(x) \sim x^{-\frac{1}{2 s}}$ and $v^{\prime \prime}(x) \sim x^{-1-\frac{1}{2 s}}$. We see that $x\left(v^{\prime}(x)\right)^{2} \sim x^{1-\frac{1}{s}}$ dominates $x^{2} v^{\prime \prime}(x) \sim x^{1-\frac{1}{2 s}}$ near $x=0$.

Remark 3.4. Note that $H_{2} \in C^{1}([0,+\infty) \times \mathbb{R})$ and that for any $p \in \mathbb{R}, x \mapsto H_{2}(x, p)+$ $\frac{s}{s-1} k^{1-1 / s} x^{1-1 / s}$ is nondecreasing with respect to $x$.

Theorem 3.4. There exists a unique nonincreasing viscosity solution $v \in \mathrm{BUC}([0,+\infty))$ of (48), in the sense of Definition 3.1.

Proof. Since we look for a nonincreasing function $v$, we first modify the equation as follows:

$$
0=\alpha v+\widetilde{H}\left(x, v^{\prime}\right)=\frac{s}{1-s} k^{1-1 / s} x^{1-1 / s},
$$

where $\widetilde{H}(x, p)=H_{1}(x, p)+\widetilde{H}_{2}(x, p)$ and $\widetilde{H}_{2}(x, p)=H_{2}\left(x, p 1_{\{p \leq 0\}}\right)$. From Remark 3.4, $\widetilde{H}_{2} \in$ $C([0,+\infty) \times \mathbb{R})$ and for any $p \in \mathbb{R}, x \mapsto \widetilde{H}_{2}(x, p)+\frac{s}{s-1} k^{1-1 / s} x^{1-1 / s}$ is nondecreasing with respect to $x$.

One can check that if $v \in \mathrm{BUC}([0,+\infty))$ is a nonincreasing viscosity solution of $(48)$, then it is a viscosity solution of (49).

Existence and uniqueness for (49) will stem from Propositions 3.2 and 3.3, once we have checked that $\widetilde{H}$ satisfies Assumptions $\left(\mathbf{H}_{\mathbf{0}}\right)-\left(\mathbf{H}_{\mathbf{4}}\right)$ with $m=2$.

- It is clear that $x \mapsto \widetilde{H}(x, 0)$ is in $\operatorname{BUC}([0,+\infty))$ and that $\widetilde{H}(x, 0)=0$.

- If $p>0$, then $\widetilde{H}_{2}(x, p)=H_{2}(x, 0)$ is a function in $\operatorname{BUC}([0,+\infty))$ that vanishes at 0 . If $p \leq 0$ and $c-p>(k x)^{-1 / s}$, then $-\frac{k}{1-s} x(c-p) \leq-\frac{1}{1-s}(c-p)^{1-s} \leq \widetilde{H}_{2}(x, p) \leq \frac{s}{1-s} k^{1-1 / s} x^{1-1 / s} \leq$ $\frac{k s}{1-s} x(c-p)$. If $p \leq 0$ and $c-p \leq(k x)^{-1 / s}$, then $k x \leq c^{-s}$, and $\widetilde{H}_{2}(x, p)=k x(p-c)$. Finally, if $x>c^{-s} / k$, then $\widetilde{H}_{2}(x, p) \leq \frac{s}{1-s} c^{1-s}$. Combining the preceding observations, we deduce that there exists a nonnegative function $\eta \in \mathrm{BUC}([0,+\infty))$ with $\eta(0)=0$ and $\zeta>0$, such that

$$
-\eta(x)-\zeta x|p| \leq \widetilde{H}_{2}(x, p) \leq \eta(x)+\zeta x 1_{x \leq c^{-s} / k}|p| .
$$

This implies that $\widetilde{H}$ satisfies $\left(\mathbf{H}_{\mathbf{1}}\right)$ with $m=2$. 
- We claim that $\widetilde{H}_{2}$ satisfies Assumption $\left(\mathbf{H}_{\mathbf{2}}\right)$ with $m=1$. Consider $p \leq 0$ : if $c-p>$ $(k x)^{-1 / s}$ and $c-p>(k y)^{-1 / s}$ then $\left|\widetilde{H}_{2}(x, p)-\widetilde{H}_{2}(y, p)\right| \leq k \max \left((k x)^{-1 / s},(k y)^{-1 / s}\right) \mid x-$ $y|\leq k(c-p)| x-y \mid$. If $c-p \leq(k x)^{-1 / s}$ and $c-p \leq(k y)^{-1 / s}$ then $\left|\widetilde{H}_{2}(x, p)-\widetilde{H}_{2}(y, p)\right|=$ $k|x-y||c-p|$. Finally, if $c-p \leq(k x)^{-1 / s}$ and $c-p \geq(k y)^{-1 / s}$, there exists $z$ between $x$ and $y$ such that $c-p=(k z)^{-1 / s}$, and $\left|\widetilde{H}_{2}(x, p)-\widetilde{H}_{2}(y, p)\right| \leq\left|\widetilde{H}_{2}(x, p)-\widetilde{H}_{2}(z, p)\right|+$ $\left|\widetilde{H}_{2}(z, p)-\widetilde{H}_{2}(y, p)\right| \leq k|x-y||c-p|$. Finally, if $p>0$, then $\left|\widetilde{H}_{2}(x, p)-\widetilde{H}_{2}(y, p)\right|=$ $\left|\widetilde{H}_{2}(x, 0)-\widetilde{H}_{2}(y, 0)\right| \leq k c|x-y|$. The claim is proved. This implies that $\widetilde{H}$ satisfies (30) in Assumption $\left(\mathbf{H}_{2}\right)$ with $m=2$. Furthermore, (31) is satisfied if $c-p x^{-1 / 2} \leq(k x)^{-1 / s}$ and $c-p y^{-1 / 2} \leq(k y)^{-1 / s}$.

- We claim that $\widetilde{H}_{2}$ satisfies Assumption $\left(\mathbf{H}_{\mathbf{3}}\right)$. Set $\tilde{p}=p 1_{\{p \leq 0\}}$ and $\tilde{q}=q 1_{\{q \leq 0\}} ;$ if $c-\tilde{p}>$ $(k x)^{-1 / s}$ and $c-\tilde{q}>(k x)^{-1 / s}\left|\widetilde{H}_{2}(x, p)-\widetilde{H}_{2}(x, q)\right|=\frac{1}{1-s}\left|(c-\tilde{p})^{1-s}-(c-\tilde{q})^{1-s}\right| \leq$ $\max \left((c-\tilde{p})^{-s},(c-\tilde{q})^{-s}\right)|\tilde{p}-\tilde{q}| \leq k x|\tilde{p}-\tilde{q}| \leq k x|p-q|$. If $c-\tilde{p} \leq(k x)^{-1 / s}$ and $c-\tilde{q} \leq$ $(k x)^{-1 / s}$ then $|\widetilde{H}(x, p)-\widetilde{H}(x, q)|=k x|\tilde{p}-\tilde{q}| \leq k x|p-q|$. Finally, if $c-\tilde{p} \leq(k x)^{-1 / s}$ and $c-\tilde{q}>(k x)^{-1 / s}$, there exists $r$ between $\tilde{p}$ and $\tilde{q}$ such that $c-r=(k x)^{-1 / s}$, and this yields that $|\widetilde{H}(x, p)-\widetilde{H}(x, q)| \leq k x|\tilde{p}-\tilde{q}| \leq k x|p-q|$. The claim is proved. It implies that $\widetilde{H}$ satisfies Assumption $\left(\mathbf{H}_{3}\right)$.

- There remains to study $\left(\mathbf{H}_{\mathbf{4}}\right)$. From (50), we see that there exists $\bar{p}>0$ and $C>0$ such that $\widetilde{H}(x, p)+C x$ is nondecreasing with respect to $x$ in $[0, \delta] \times\{|p| \geq \bar{p}\}$, because the dominating behavior is that of $\beta x|p|^{m}$ in this region. Let $\chi$ be a smooth monotone function on $\mathbb{R}_{+}$such that $\chi(t)=1$ for $t \geq 2 \bar{p}$ and $\chi(t)=0$ for $t \leq \bar{p}$. We can split $\widetilde{H}$ as follows: $\widetilde{H}(x, p)=\widetilde{H}_{3}(x, p)+\widetilde{H}_{4}(x, p)$ where $\widetilde{H}_{3}(x, p)=\chi(|p|) \widetilde{H}(x, p)+C x$ and $\widetilde{H}_{4}(x, p)=(1-\chi(|p|)) \widetilde{H}(x, p)-C x$, which proves that $\widetilde{H}$ satisfies $\left(\mathbf{H}_{4}\right)$.

Even if (31) may not hold for every $x, y$ and $p$, Theorem 3.3 holds for $\widetilde{H}$. The modifications in the proof arise in estimates (43) and (45). We focus on (45) and briefly sketch the required modification that should be implemented after (44).

If $c-q_{\epsilon} x_{\epsilon}^{-1 / 2} \leq\left(k x_{\epsilon}\right)^{-1 / s}$ and $c-q_{\epsilon}\left(y_{\epsilon}+\sqrt{\epsilon}\right)^{-1 / 2} \leq\left(k\left(y_{\epsilon}+\sqrt{\epsilon}\right)\right)^{-1 / s}$, we can use (31) and the proof follows as for Theorem 3.3. Note also that the case when $q_{\epsilon} \geq 0$ is easily dealt with.

Let us therefore focus on the case when $q_{\epsilon}<0$, i.e. $x_{\epsilon}<y_{\epsilon}+\sqrt{\epsilon}$, and both $c-q_{\epsilon} x_{\epsilon}^{-1 / 2}>\left(k x_{\epsilon}\right)^{-1 / s}$ and $c-q_{\epsilon}\left(y_{\epsilon}+\sqrt{\epsilon}\right)^{-1 / 2}>\left(k\left(y_{\epsilon}+\sqrt{\epsilon}\right)\right)^{-1 / s}$ : since $q_{\epsilon}=o\left(\epsilon^{-1 / 2}\right)$ and $s<1$, we see that $x_{\epsilon}^{-1 / 2}=o\left(\epsilon^{-1 / 2}\right)$. We also know that $\left(y_{\epsilon}+\sqrt{\epsilon}\right)^{\frac{1}{2}}-\left(x_{\epsilon}\right)^{\frac{1}{2}}=o(\sqrt{\epsilon})$. From these observations, we can see that

$$
\begin{aligned}
\left|\left(c-q_{\epsilon} x_{\epsilon}^{-1 / 2}\right)^{1-s}-\left(c-q_{\epsilon}\left(y_{\epsilon}+\sqrt{\epsilon}\right)^{-1 / 2}\right)^{1-s}\right| & \left.\leq C\left|q_{\epsilon}\right|\left(y_{\epsilon}+\sqrt{\epsilon}\right)^{1 / 2}-x_{\epsilon}^{1 / 2}\right)\left(\frac{y_{\epsilon}+\sqrt{\epsilon}}{x_{\epsilon}}\right)^{1 / 2} \\
& \rightarrow 0 \text { as } \epsilon \rightarrow 0 .
\end{aligned}
$$

On the other hand, $\left(k x_{\epsilon}\right)^{1-\frac{1}{s}}-\left(k\left(y_{\epsilon}+\sqrt{\epsilon}\right)\right)^{1-\frac{1}{s}} \geq 0$. Therefore $\liminf \operatorname{in}_{\epsilon \rightarrow 0} \widetilde{H}_{2}\left(x_{\epsilon}, q_{\epsilon} x_{\epsilon}^{-1 / 2}\right)-$ $\widetilde{H}_{2}\left(y_{\epsilon}+\sqrt{\epsilon}, q_{\epsilon}\left(y_{\epsilon}+\sqrt{\epsilon}\right)^{-1 / 2}\right) \geq 0$, and the proof follows as for Theorem 3.3 .

The case when either $c-q_{\epsilon} x_{\epsilon}^{-1 / 2}>\left(k x_{\epsilon}\right)^{-1 / s}$ and $c-q_{\epsilon}\left(y_{\epsilon}+\sqrt{\epsilon}\right)^{-1 / 2} \leq\left(k\left(y_{\epsilon}+\sqrt{\epsilon}\right)\right)^{-1 / s}$ or $c-q_{\epsilon} x_{\epsilon}^{-1 / 2} \leq\left(k x_{\epsilon}\right)^{-1 / s}$ and $c-q_{\epsilon}\left(y_{\epsilon}+\sqrt{\epsilon}\right)^{-1 / 2}>\left(k\left(y_{\epsilon}+\sqrt{\epsilon}\right)\right)^{-1 / s}$ can be handled by introducing $z_{\epsilon}$ between $x_{\epsilon}$ and $y_{\epsilon}+\sqrt{\epsilon}$ such that $c-q_{\epsilon} z_{\epsilon}^{-1 / 2}=\left(k z_{\epsilon}\right)^{-1 / s}$.

Finally, we obtain that all the results in $\S 3.1$ and 3.2 hold and that (49) has a unique solution $v \in \operatorname{BUC}([0,+\infty))$. This implies that there is at most one nonincreasing viscosity solution $v \in \operatorname{BUC}([0,+\infty))$ of $(48)$.

We now need to prove that the function $v$ that we just found is indeed nonincreasing: Since 
$x \mapsto \widetilde{H}(x, p)+\frac{s}{s-1} k^{1-1 / s} x^{1-1 / s}$ is nondecreasing with respect to $x$, we see that for $h>0$, $x \mapsto v(x+h)$ is a sub solution of (49).

This implies that the viscosity solution $v$ of (49) is nonincreasing. Hence $v$ is the nonincreasing BUC viscosity solution of (48).

Remark 3.5. The same strategy could be applied if $H_{1}(x, p)=\beta x p+\gamma x p^{2}$ provided $\alpha+\beta>0$, but it would be more difficult to prove that the solution of (49) is nonincreasing.

\section{Extension: a closed industry with two technologies}

Suppose now that there are two types of production units (different technologies), with different prospection and production costs. The indices $i=1,2$ will be used to distinguish the two kinds of production units.

- The total reserve of type $i$ will be noted $R_{i}(t) \in \mathbb{R}_{+}$. With the assumption above, $R_{i}(t)$ can also be viewed as the quantity of production units of type $i$.

- It is assumed that the production capacity is proportional to the reserve. Let $k, 0<k<1$ be the production capacity of a single production unit (with reserve 1): to begin with, $k$ is assumed to be independent of the reserve type $i=1,2$.

- Let $c_{i}>0$ be the unitary production cost (i.e. the production cost of a unit of ore) of the industry of type $i$, with $c_{1}<c_{2}$.

- Each production unit of type $i$ can invest into prospection. For the industry of type $i$, the flux invested into prospection by a single unit is $\alpha_{i} d t$ ( $\alpha_{i}$ is a control parameter). An investment rate of $\alpha_{i}$ increases the reserves of type $j, j=1,2$ with a rate of $\phi_{i, j}\left(\alpha_{i}\right)$, where $\phi_{i, j}$ are increasing and concave functions on $\mathbb{R}_{+}$with $\phi_{i, j}(0)=0$. To begin with, it is possible to assume that $\phi_{i, j}=0$ if $i \neq j$, i.e. the new reserves created by the industry of type $i$ are only of type $i$. For example, one may choose $\phi_{i}(\alpha)=\sqrt{\alpha}$

- The other control parameters are the production rates $\beta_{i}$ of a unit of type $i, i=1,2$, with $0 \leq \beta_{i} \leq k$.

- The discount factor of the expected income is $r_{i}>0$ for the industry of type $i$.

\subsection{The strategy of the production units}

Let $u_{i}\left(R_{1}, R_{2}, X\right)$ be the expected benefit of a production unit of type $i$, discounted by $r_{i}$. As above, when a production unit of type $i$ produces $q$ units of ore, its production cost is $q c_{i}$ and the cost of decreasing the reserves is $q u_{i}\left(R_{1}, R_{2}, X\right)$. The total cost is $q c_{i}+q u_{i}\left(R_{1}, R_{2}, X\right)$ and the income is $p q$. Therefore, the following inequality should hold: $p \geq c_{i}+u_{i}\left(R_{1}, R_{2}, X\right)$. If $p=c_{i}+u_{i}\left(R_{1}, R_{2}, X\right)$, it is indifferent for a unit of type $i$ to produce or not.

Fixing the price $p$ and the global productions $Q_{i}, i=1,2$ knowing $u_{i}\left(R_{1}, R_{2}, X\right)$ As above, the price $p$ and global productions $Q_{i}$ can be found by matching offer and demand. Let $P^{*}\left(R_{1}, R_{2}, X\right)$ be the price function. The cash income for a unit of ore produced by the industry of type $i$ is $g_{i}\left(R_{1}, R_{2}, X, u_{1}, u_{2}\right)=P^{*}\left(R_{1}, R_{2}, X, u_{1}, u_{2}\right)-c_{i}$.

In order to divide the number of cases by two, we assume that $u_{2}+c_{2}>u_{1}+c_{1}$, but should the opposite case occur, we would compute the prices and productions in a symmetric way, by exchanging the indices 1 and 2. If $u_{2}+c_{2}>u_{1}+c_{1}$, there are four different cases: 
1. The two industries produce at full capacity when $p>u_{2}+c_{2}$.

The total productions are $Q_{1}=k R_{1}$ and $Q_{2}=k R_{2}$. Matching offer and demand yields $k\left(R_{1}+R_{2}\right)=X \tilde{D}(p)$, i.e $P^{*}\left(R_{1}, R_{2}, X, u_{1}, u_{2}\right)=\tilde{D}^{-1}\left(k\left(\frac{R_{1}}{X}+\frac{R_{2}}{X}\right)\right)$. The inequality is then equivalent to $\tilde{D}^{-1}\left(k\left(\frac{R_{1}}{X}+\frac{R_{2}}{X}\right)\right)>\max \left(u_{1}+c_{1}, u_{2}+c_{2}\right)$. In this regime, $g_{i}\left(R_{1}, R_{2}, X, u_{1}, u_{2}\right)=\tilde{D}^{-1}\left(k\left(\frac{R_{1}}{X}+\frac{R_{2}}{X}\right)\right)-c_{i}$.

2. Industry 1 produces at full capacity and industry 2 has a positive but partial production when $u_{1}+c_{1}<p=u_{2}+c_{2}$. The total productions are $Q_{1}=k R_{1}$ and $Q_{2}$ is obtained by matching offer and demand: $Q_{2} / X=\tilde{D}\left(c_{2}+u_{2}\right)-k R_{1} / X$. Then $k R_{2}>Q_{2}>0$ occurs if an only if $\tilde{D}^{-1}\left(k\left(\frac{R_{1}}{X}+\frac{R_{2}}{X}\right)\right)<u_{2}+c_{2}<\tilde{D}^{-1}\left(k \frac{R_{1}}{X}\right)$. In this regime, $g_{1}\left(R_{1}, R_{2}, X, u_{1}, u_{2}\right)=u_{2}+c_{2}-c_{1}$ and $g_{2}\left(R_{1}, R_{2}, X, u_{1}, u_{2}\right)=u_{2}$.

3. Industry 1 produces at full capacity and industry 2 does not produce when $u_{1}+c_{1}<p<u_{2}+c_{2}$. The total productions are $Q_{1}=k R_{1}$ and $Q_{2}=0$; matching offer and demand yields $P^{*}\left(R_{1}, R_{2}, X, u_{1}, u_{2}\right)=\tilde{D}^{-1}\left(k \frac{R_{1}}{X}\right)$. The inequality is equivalent to $u_{2}+c_{2}>\tilde{D}^{-1}\left(k \frac{R_{1}}{X}\right)>u_{1}+c_{1}$. In this regime, $g_{i}\left(R_{1}, R_{2}, X, u_{1}, u_{2}\right)=\tilde{D}^{-1}\left(k \frac{R_{1}}{X}\right)-c_{i}$.

4. Industry 1 has a positive but partial production and industry 2 does not produce when $u_{1}+c_{1}=p<u_{2}+c_{2}$. Then $Q_{2}=0$ and matching offer and demand yields $Q_{1} / X=\tilde{D}\left(c_{1}+u_{1}\right)$. In this regime, $u_{2}+c_{2}>u_{1}+c_{1}>\tilde{D}^{-1}\left(k \frac{R_{1}}{X}\right)$, $g_{1}\left(R_{1}, R_{2}, X, u_{1}, u_{2}\right)=u_{1}$ and $g_{2}\left(R_{1}, R_{2}, X, u_{1}, u_{2}\right)=u_{1}+c_{1}-c_{2}$.

Summarizing, if $u 1+c 1<u_{2}+c_{2}$, then the total productions are given by the continuous functions

$$
\begin{aligned}
& Q_{1}^{*}\left(R_{1}, R_{2}, X, u_{1}, u_{2}\right)=X \min \left(\frac{k R_{1}}{X}, \tilde{D}\left(c_{1}+u_{1}\right)\right) \\
& Q_{2}^{*}\left(R_{1}, R_{2}, X, u_{1}, u_{2}\right)=X \max \left(0, \min \left(\frac{k R_{2}}{X}, \tilde{D}\left(c_{2}+u_{2}\right)-\frac{k R_{1}}{X}\right)\right)
\end{aligned}
$$

The optimal strategy of a production unit As above, the expected values $u_{i}$ are obtained by optimizing on the controls, knowing the dynamics of $R_{1}$ and $R_{2}$ :

$$
\begin{aligned}
& u_{i}\left(R_{1}, R_{2}, X\right) \\
= & \left(1-r_{i} d t\right) \max _{\alpha_{i}>0,0 \leq \beta_{i} \leq k} \mathbb{E}\left(\begin{array}{l}
\left(\beta_{i} g_{i}\left(R_{1}, R_{2}, X, u_{1}, u_{2}\right)-\alpha_{i}\right) d t \\
+\left(1+\phi_{i}\left(\alpha_{i}\right) d t-\beta_{i} d t\right) u_{i}\left(R_{1}+d R_{1}, R_{2}+d R_{2}, X+d X\right)
\end{array}\right)
\end{aligned}
$$

\subsection{Partial differential equations}

A first order expansion in (53) and the equilibrium relations $d R_{i}=\left(R_{i} \phi_{i}\left(\alpha_{i}^{*}\right)-Q_{i}^{*}\left(R_{1}, R_{2}, X, u_{1}, u_{2}\right)\right) d t$, where $\alpha_{i}^{*}$ is the optimal value of $\alpha_{i}$, yield the system of partial differential equations: for $i=1,2$,

$$
\begin{aligned}
0= & -r_{i} u_{i}+k\left(g_{i}\left(\cdot, u_{1}, u_{2}\right)-u_{i}\right)-Q_{1}^{*}\left(\cdot, u_{1}, u_{2}\right) \partial_{R_{1}} u_{i}-Q_{2}^{*}\left(\cdot, u_{1}, u_{2}\right) \partial_{R_{2}} u_{i} \\
& +\partial_{R_{i}}\left(R_{i} \max _{\alpha_{i}}\left(u_{i} \phi_{i}\left(\alpha_{i}\right)-\alpha_{i}\right)\right)+\phi_{j}\left(\alpha_{j}^{*}\right) R_{j} \partial_{R_{j}} u_{i}+b X \partial_{X} u_{i}+\frac{1}{2} \sigma^{2} X^{2} \partial_{X X} u_{i},
\end{aligned}
$$

where $j=2$ (resp. $j=1$ ) if $i=1$ (resp. $i=2$ ). 
Homogeneity: reduced variables Observe that $g_{i}$ and $\tilde{Q}_{i}^{*}=Q_{i}^{*} / X$ are functions of $y_{1}=$ $R_{1} / X, y_{2}=R_{2} / X, u_{1}$ and $\left.u_{2}\right)$. It is natural to look for a solution of the form $u_{i}\left(R_{1}, R_{2}, X\right)=$ $v_{i}\left(y_{1}, y_{2}\right)$; this leads to the following system:

$$
\begin{aligned}
0= & -r_{1} v_{1}+k\left(g_{1}\left(y_{1}, y_{2}, v_{1}, v_{2}\right)-v_{1}\right)-\tilde{Q}_{1}^{*}\left(y_{1}, y_{2}, v_{1}, v_{2}\right) \partial_{y_{1}} v_{1}-\tilde{Q}_{2}^{*}\left(y_{1}, y_{2}, v_{1}, v_{2}\right) \partial_{y_{2}} v_{1} \\
& +\partial_{y_{1}}\left(y_{1} \max _{\alpha_{1}}\left(v_{1} \phi_{1}\left(\alpha_{1}\right)-\alpha_{1}\right)\right)+\phi_{2}\left(\alpha_{2}^{*}\right) y_{2} \partial_{y_{2}} v_{1} \\
& +\left(\sigma^{2}-b\right)\left(y_{1} \partial_{y_{1}} v_{1}+y_{2} \partial_{y_{2}} v_{1}\right)+\frac{1}{2} \sigma^{2}\left(y_{1}^{2} \partial_{y_{1}}^{2} v_{1}+2 y_{1} y_{2} \partial_{y_{1} y_{2}}^{2} v_{1}+y_{2}^{2} \partial_{y_{2}}^{2} v_{1}\right) \\
0 & -r_{2} v_{2}+k\left(g_{2}\left(y_{1}, y_{2}, v_{1}, v_{2}\right)-v_{2}\right)-\tilde{Q}_{1}^{*}\left(y_{1}, y_{2}, v_{1}, v_{2}\right) \partial_{y_{1}} v_{2}-\tilde{Q}_{2}^{*}\left(y_{1}, y_{2}, v_{1}, v_{2}\right) \partial_{y_{2}} v_{2} \\
& +\partial_{y_{2}}\left(y_{2} \max _{\alpha_{2}}\left(v_{2} \phi_{2}\left(\alpha_{2}\right)-\alpha_{2}\right)\right)+\phi_{1}\left(\alpha_{1}^{*}\right) y_{1} \partial_{y_{1}} v_{2} \\
& +\left(\sigma^{2}-b\right)\left(y_{1} \partial_{y_{1}} v_{2}+y_{2} \partial_{y_{2}} v_{2}\right)+\frac{1}{2} \sigma^{2}\left(y_{1}^{2} \partial_{y_{1}}^{2} v_{2}+2 y_{1} y_{2} \partial_{y_{1} y_{2}}^{2} v_{2}+y_{2}^{2} \partial_{y_{2}}^{2} v_{2}\right)
\end{aligned}
$$

A Hamilton-Jacobi equation In the case when $r_{1}=r_{2}=r$, consider the degenerate second order Hamilton-Jacobi equation:

$$
(b-r) V-H\left(y_{1}, y_{2}, D V\right)-b\left(y_{1} \partial_{y_{1}} V+y_{2} \partial_{y_{2}} V\right)+\frac{\sigma^{2}}{2}\left(y_{1}^{2} \partial_{y_{1}}^{2} V+2 y_{1} y_{2} \partial_{y_{1} y_{2}}^{2} V+y_{2}^{2} \partial_{y_{2}}^{2} V\right)=0
$$

where $H\left(y_{1}, y_{2}, v_{1}, v_{2}\right)=H_{1}\left(y_{1}, y_{2}, v_{1}, v_{2}\right)+H_{2}\left(y_{1}, y_{2}, v_{1}, v_{2}\right)$ and

$$
H_{2}\left(y_{1}, y_{2}, v_{1}, v_{2}\right)=-\sum_{i=1,2} y_{i} \max _{\alpha_{i} \geq 0}\left(\phi_{i}\left(\alpha_{i}\right) v_{i}-\alpha_{i}\right) .
$$

We give the expression of $H_{1}$ when $v_{1}+c_{1}<v_{2}+c_{2}$. In the opposite case, it is enough to switch the indices $i=1,2$.

$$
=\left\{\begin{array}{rr}
k\left(y_{1}\left(v_{1}+c_{1}\right)+y_{2}\left(v_{2}+c_{2}\right)-\left(y_{1}+y_{2}\right) \tilde{D}^{-1}\left(k\left(y_{1}+y_{2}\right)\right)\right)-\int_{D^{-1}\left(k\left(y_{1}+y_{2}\right)\right)}^{M} & \tilde{D}(z) d z \\
k y_{1}\left(v_{1}+c_{1}-v_{2}-c_{2}\right)-\int_{v_{2}+c_{2}}^{M} \tilde{D}(z) d z & \text { if } D^{-1}\left(k\left(y_{1}+y_{2}\right)\right)>c_{2}, \\
k y_{1}\left(v_{1}+c_{1}-\tilde{D}^{-1}\left(k y_{1}\right)\right)-\int_{D^{-1}\left(k y_{1}\right)}^{M} \tilde{D}(z) d z & \text { if } D^{-1}\left(k\left(y_{1}+y_{2}\right)\right)<v_{2}+c_{2}<D^{-1}\left(k y_{1}\right), \\
-\int_{v_{1}+c_{1}}^{M} \tilde{D}(z) d z & \text { if } v_{1}+c_{1}<D^{-1}\left(k y_{1}\right)<v_{2}+c_{2},
\end{array}\right.
$$

The Hamiltonians $H_{1}$ and $H_{2}$ have the following monotonicity property:

$$
\left(H_{k, p}(y, p)-H_{k, p}(z, q), p-q\right)-\left(H_{k, y}(y, p)-H_{k, y}(z, q), y-z\right) \leq 0, \quad k=1,2 .
$$


If $r_{1}=r_{2}=r$ and $V$ is a solution of (56) such that $\partial_{1} V \geq 0$ and $\partial_{2} V \geq 0$, then $\left(v_{1}, v_{2}\right)=D V$ is a solution to (55) with nonnegative components.

When $D$ is given by $(22)$, the Hamiltonian $H_{1}\left(\cdot, v_{1}, v_{2}\right)$ has the following form if $v_{1}+c_{1}<v_{2}+c_{2}$ :

$$
H_{1}\left(y_{1}, y_{2}, v_{1}, v_{2}\right)=\left\{\begin{array}{r}
k y_{1}\left(v_{1}+c_{1}\right)+k y_{2}\left(v_{2}+c_{2}\right)+\frac{s}{1-s} k^{1-\frac{1}{s}}\left(y_{1}+y_{2}\right)^{1-\frac{1}{s}} \\
\text { if }\left(k\left(y_{1}+y_{2}\right)\right)^{-\frac{1}{s}}>v_{2}+c_{2}, \\
k y_{1}\left(v_{1}+c_{1}-v_{2}-c_{2}\right)+\frac{1}{1-s}\left(v_{2}+c_{2}\right)^{1-s} \\
\text { if }\left(k\left(y_{1}+y_{2}\right)\right)^{-\frac{1}{s}}<v_{2}+c_{2}<\left(k y_{1}\right)^{-\frac{1}{s}}, \\
k y_{1}\left(v_{1}+c_{1}\right)+\frac{s}{1-s} k^{1-\frac{1}{s}} y_{1}^{1-\frac{1}{s}} \\
\text { if } v_{1}+c_{1}<\left(k y_{1}\right)^{-\frac{1}{s}}<v_{2}+c_{2}, \\
\frac{1}{1-s}\left(c_{1}+v_{1}\right)^{1-s} \\
\text { if }\left(k y_{1}\right)^{-\frac{1}{s}}<v_{1}+c_{1} .
\end{array}\right.
$$

\section{Tuning the parameters}

We consider the model described in $\S 2.1$, assuming that the function $\phi$ describing the efficiency of prospection is of the form

$$
\phi(\alpha)=C \sqrt{\alpha},
$$

and that the demand function if of the form $D(X, p)=X p^{-s}$. The model therefore depends on a set $\mathcal{S}$ of seven parameters, namely

- the interest rate $r$

- the growth rate $b$ and the volatility $\sigma$ of the process $X_{t}$

- the production cost $c$ and the production capacity $k$

- the parameter $C$ in (59)

- the exponent $s$ in the demand function.

From (1), (10), the reduced variable $y_{t}=R_{t} / X_{t}$ satisfies the stochastic differential equation:

$$
d y_{t}=\Psi_{\mathcal{S}}\left(y_{t}\right) d t-\sigma y_{t} d W_{t},
$$

with the drift given by

$$
\Psi_{\mathcal{S}}(y)=\left(C v(y)-\min \left(k, \frac{1}{y(c+v(y))^{s}}\right)+\sigma^{2}-b\right) y
$$

The model must be calibrated in order to fit the data, which is, for a given material (e.g. copper, zinc, nickel, cobalt), the series of the prices every month in the last 40 years. There are thus 480 observed prices $\left(p_{i}\right)_{i=1, \ldots, 480}$, see for example Figure 1 where the price of copper is plotted. The time interval between two observations is $\Delta t=1 / 12$. Let $t_{i}=i \Delta t$ be the date of the $i^{\text {th }}$ observation. 
Let $v_{\mathcal{S}}$ be the solution to $(23)$ when the parameter set if $\mathcal{S}$. Knowing $\mathcal{S}$ and $v_{\mathcal{S}}$, we can map any observed prices $p_{i}$ to a value $y_{i}$ by inverting $p_{i}=\max \left(\left(k y_{i}\right)^{-1 / s}, v_{\mathcal{S}}\left(y_{i}\right)+c\right)$, see $(4)$.

The parameters estimation consists of maximizing the likelihood of the observations. This amounts to minimizing

$$
J(\mathcal{S})=\frac{1}{2} \sum_{i}\left(\frac{y_{i+1}-y_{i}-\Delta t \Psi_{\mathcal{S}}\left(y_{i}\right)}{\sigma y_{i} \sqrt{\Delta t}}\right)^{2}+\sum_{i} \ln \left(\sigma y_{i}\right),
$$

given a positive parameter $\epsilon$. We also impose some constraints on the parameters. The constrained minimization of $J$ is done using Broyden-Fletcher-Goldfarb-Shanno (BFGS) algorithm.

For copper, we found that the set of parameters: $r=0.18, b=0.01, c=0.2 k=0.29$, $C=1, \sigma=0.20$ and $s=0.8$ permits to fit the data in a rather satisfactory manner. In Figure 2, we plot both the distribution of the observed prices (dotted line) and the distribution of the prices predicted by the model with the above parameters. In both the observed and predicted distributions, there is narrow peak correponding to small prices at $p \approx 0.6$ and a bump for larger prices: the narrow peak corresponds to periods when the demand is low, during which $p=c+u(R, X)$ and $Q(R, X)=X /(c+u(R, X))^{s}$. The bump corresponds to periods when the demand is high, during which $p>c+u(R, X)$ and $Q(R, X)=k R$.

In Figure 3, we plot the optimal price as a function of $R / X$. The two different regimes discussed in $\S 2.1 .2$ appear clearly: in the first regime, corresponding to small values of $R / X$ (the bump in the price distribution in Figure 2) the industry produces at full capacity. In the second regime, corresponding to higher values of $R / X$, (the peaks in the price distribution in Figure 2), the price is low, and the industry has a partial production.

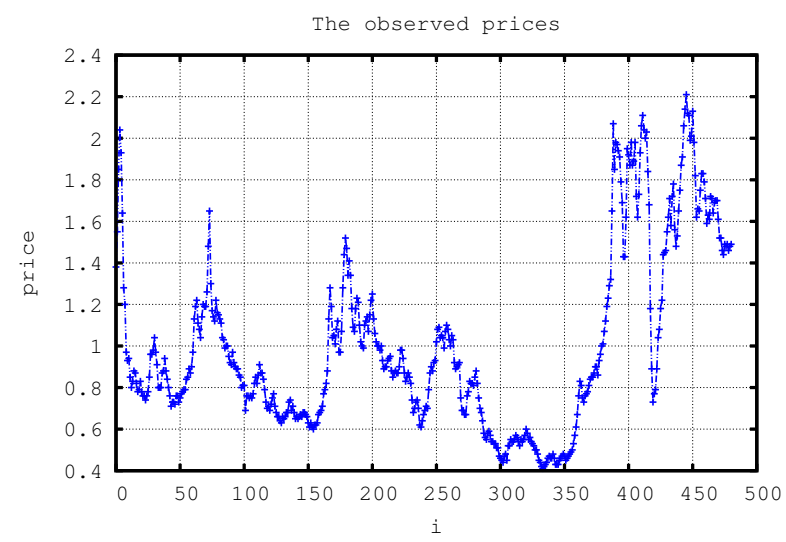

Figure 1: The observed prices of copper

We have carried the same program for several materials: in Figure 4, we compare the historical and predicted distributions of prices for zinc, with the following set of parameters: $r=0.2$, $b=0.01, c=0.2 k=0.35, C=1.05, \sigma=0.23$ and $s=0.92$ 


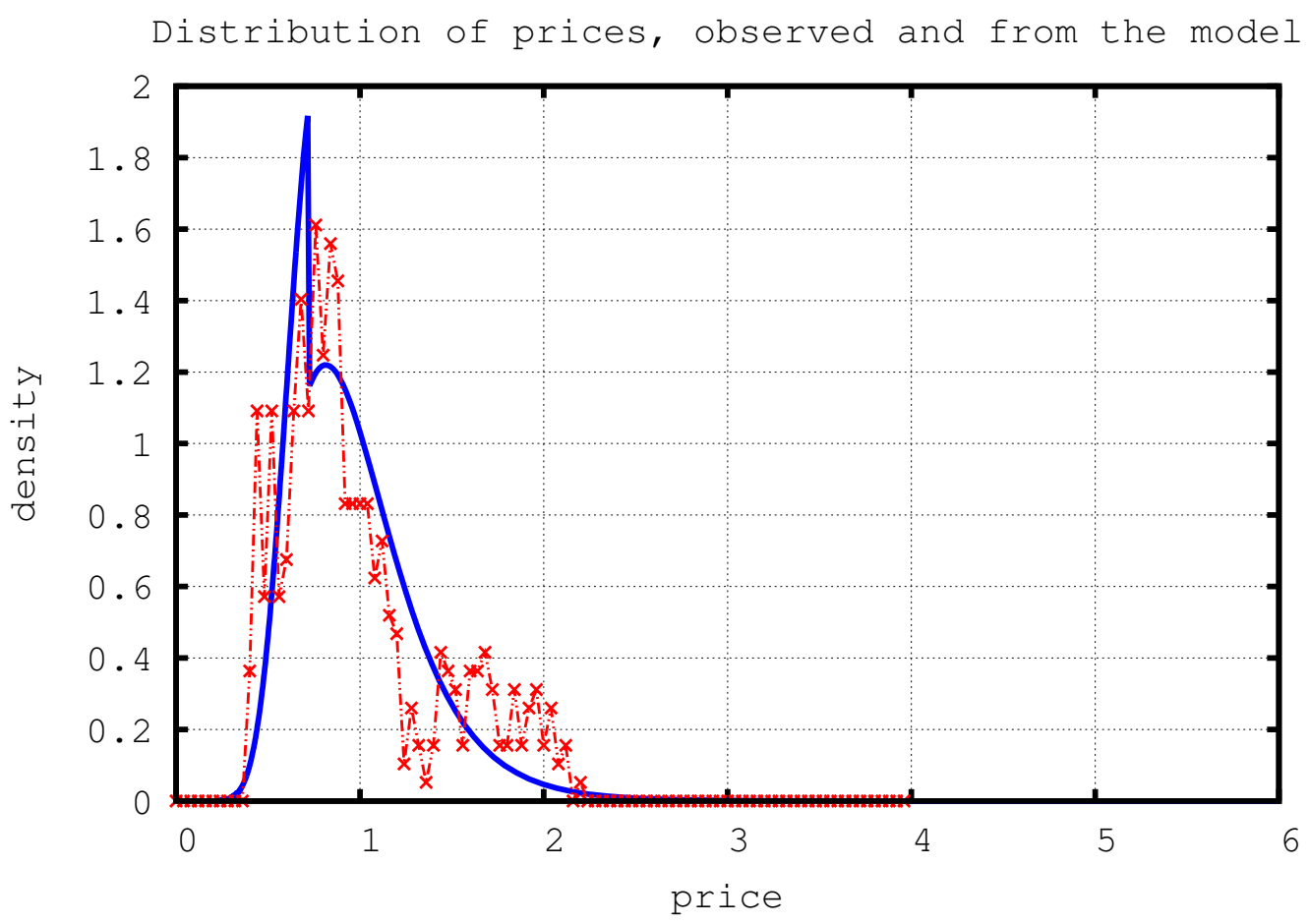

Figure 2: Copper: the distribution of the prices (observed and computed from the model)

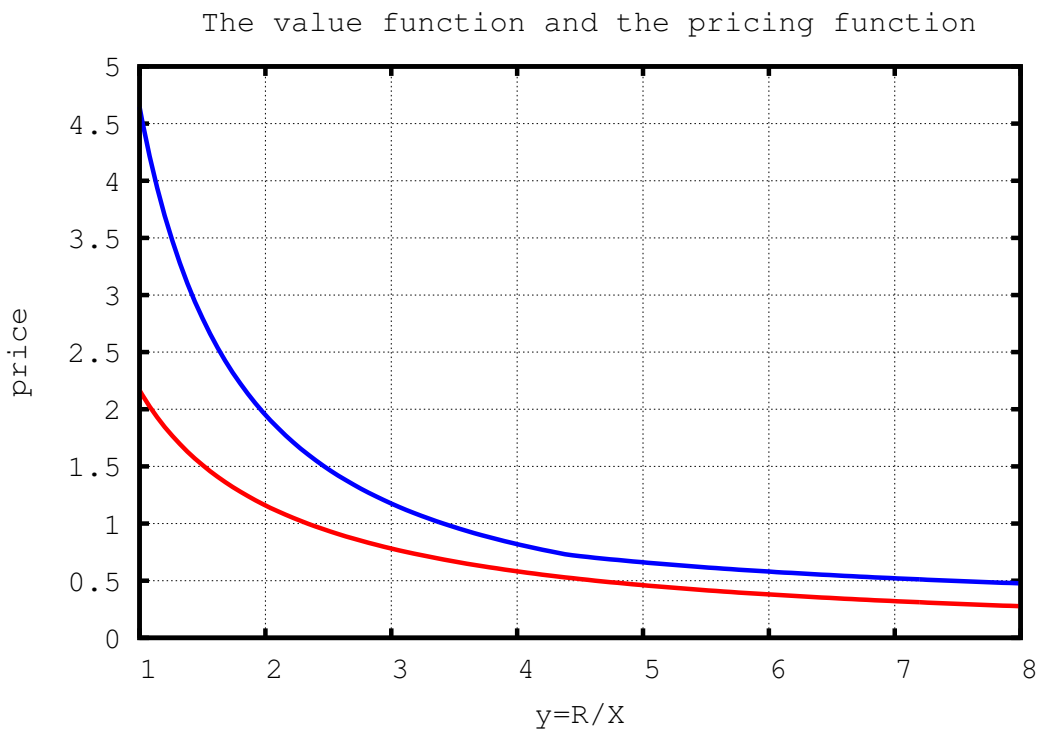

Figure 3: Copper: in red : the solution $v$ of (12); in blue: the price $P^{*}$ predicted by the model as a function of $y=R / X$. 


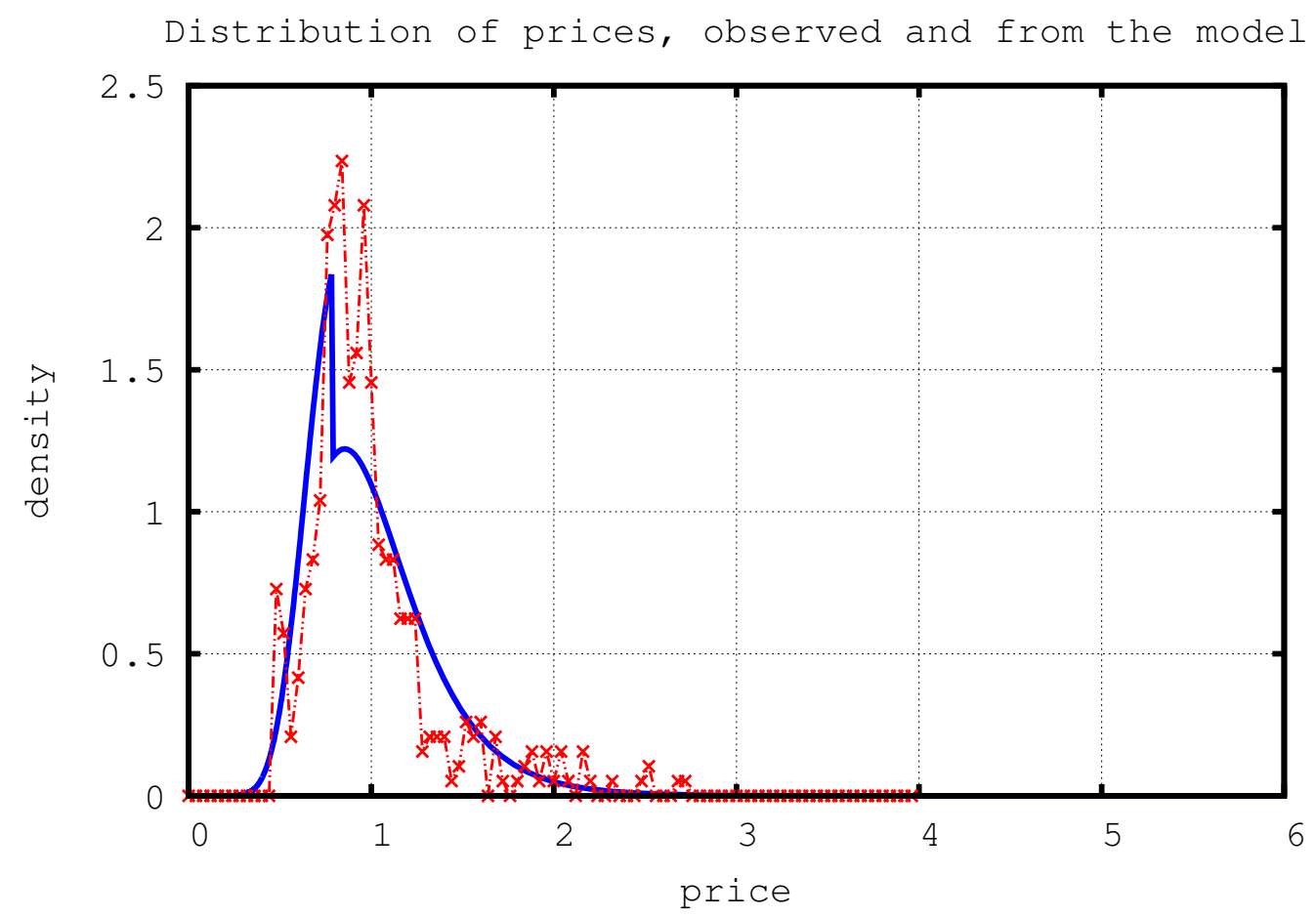

Figure 4: Zinc: the distribution of the prices (observed and computed from the model)

\section{Numerical simulations of the closed industry with two tech- nologies}

\subsection{Case 1}

We consider the model presented in $\S 4$ with

$$
\tilde{D}(p)=p^{-s}, \quad \phi_{1}(\alpha)=\phi_{2}(\alpha)=0.895 \sqrt{\alpha},
$$

with the following parameters

$$
\begin{aligned}
& r_{1}=r_{2}=0.18 ; c_{1}=0.35 ; c_{2}=0.6 \\
& k=0.3 ; s=0.6 ; \sigma=0.15 ; b=0.04
\end{aligned}
$$

In this model, the cost of production of industry 1 is smaller than that of industry $2: c_{1}<c_{2}$, whereas the investments into prospection are equally efficient.

On Figure 5, we plot the contours of $v_{1}$ and $v_{2}$ as functions of $y_{1}=R_{1} / X$ and $y_{1}=R_{2} / X$. Note that both $v_{1}$ and $v_{2}$ blow up at $(0,0)$.

On Figure 6, we plot the rescaled productions $\tilde{Q}_{1}=\frac{Q_{1}}{X}$ and $\tilde{Q}_{2}=\frac{Q_{2}}{X}$ as functions of $y_{1}=R_{1} / X$ and $y_{2}=R_{2} / X$. We plot the contours of the same functions on Figure 7. Note the region near the $y_{2}$ axis in which the production of industry 1 is zero. The distribution of agents and the price as functions of $R_{1} / X$ and $R_{2} / X$ are plotted on Figure 8.

On Figure 9, we plot the different zones corresponding to the different regimes of the Hamiltonian. The zones numbered from 0 to 3 correspond to the case when $v_{1}+c_{1} \leq v_{2}+c_{2}$ and to the four successive regimes in the definition of $H_{1}$ in (58). The zones numbered from 4 to 
7 correspond to the case when $v_{2}+c_{2} \leq v_{1}+c_{1}$ and to the four related regimes in the symmetrized version of (58). We see that all the regimes are present except the first one in the case $v_{1}+c_{1} \leq v_{2}+c_{2}$, i.e. there are seven different zones. 
contours of $\mathrm{v} 1$

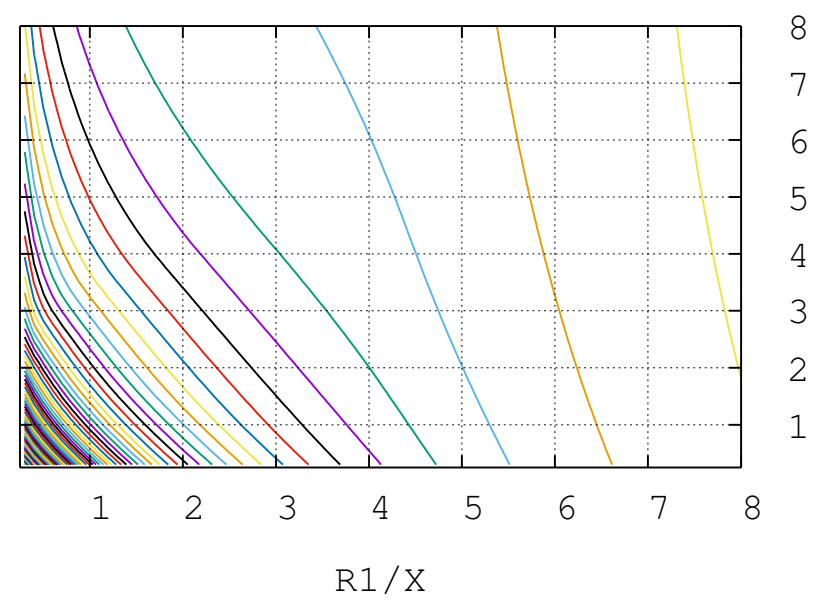

$\mathrm{R} 2 / \mathrm{X}$

contours of v2

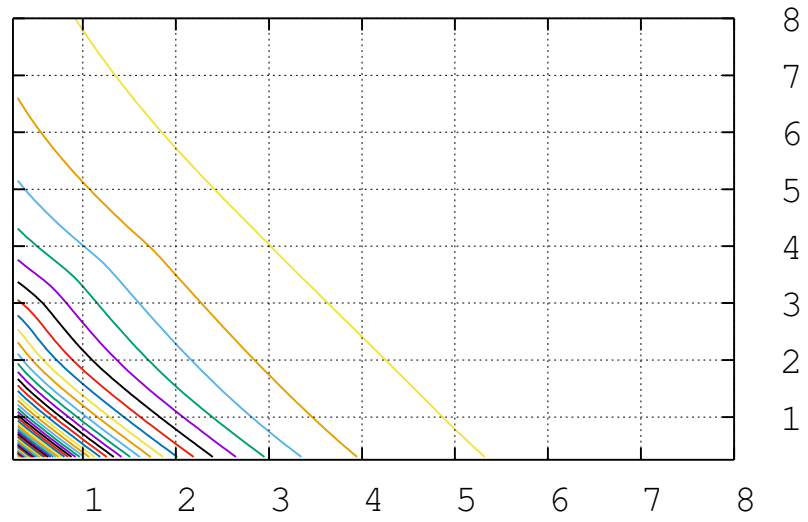

R2 / X

Figure 5: The contours of $v_{1}$ and $v_{2}$ 
production of industry 1

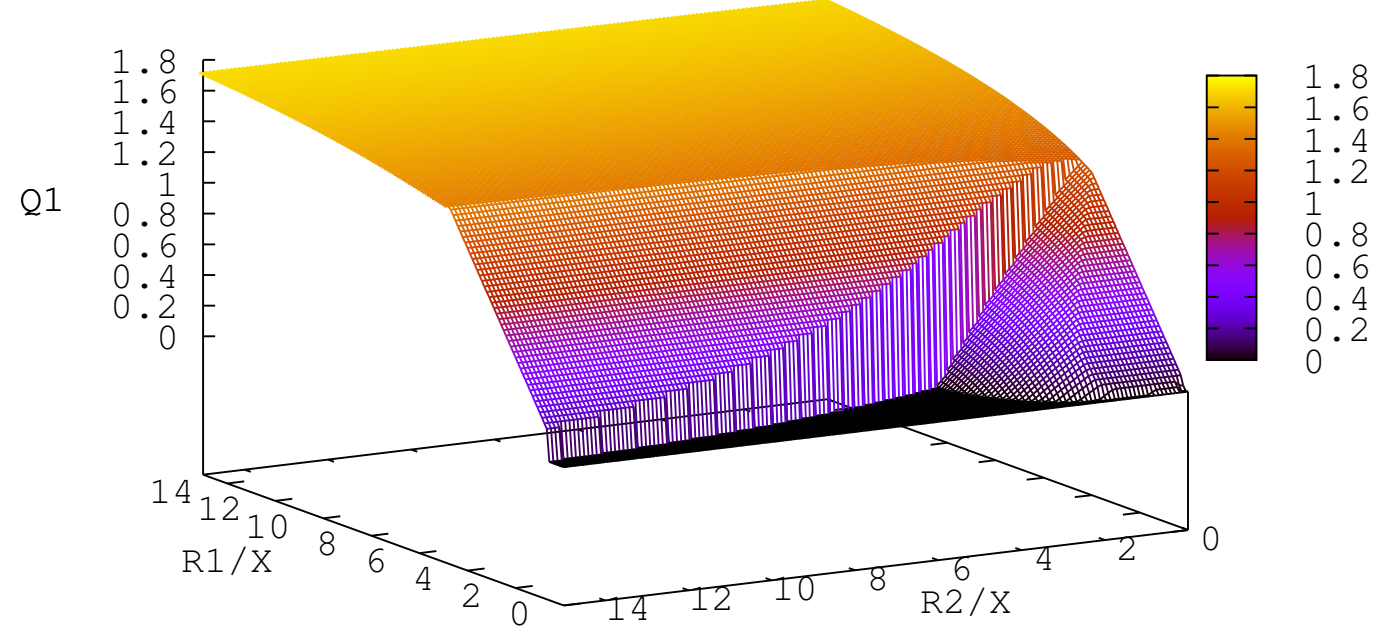

production of industry 2

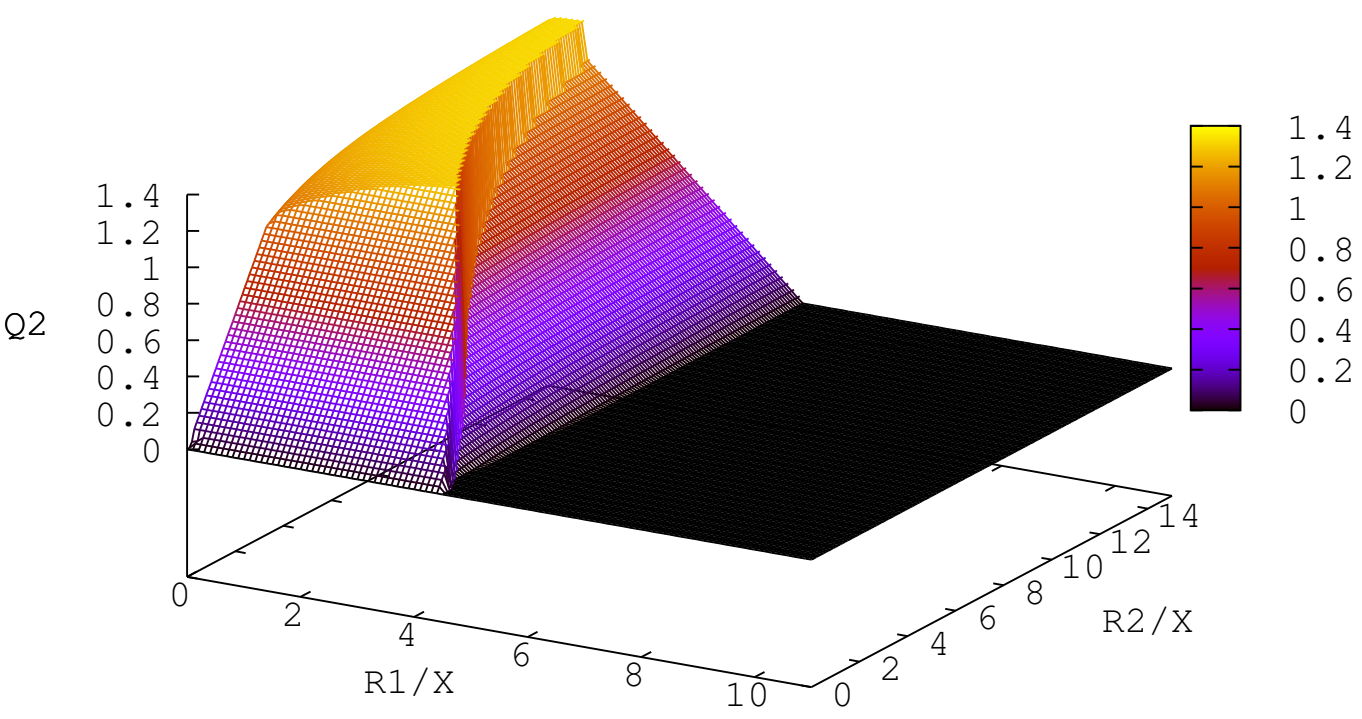

Figure 6: The productions $\tilde{Q}_{1}=\frac{Q_{1}}{X}$ and $\tilde{Q}_{2}=\frac{Q_{2}}{X}$ 
production of industry 1

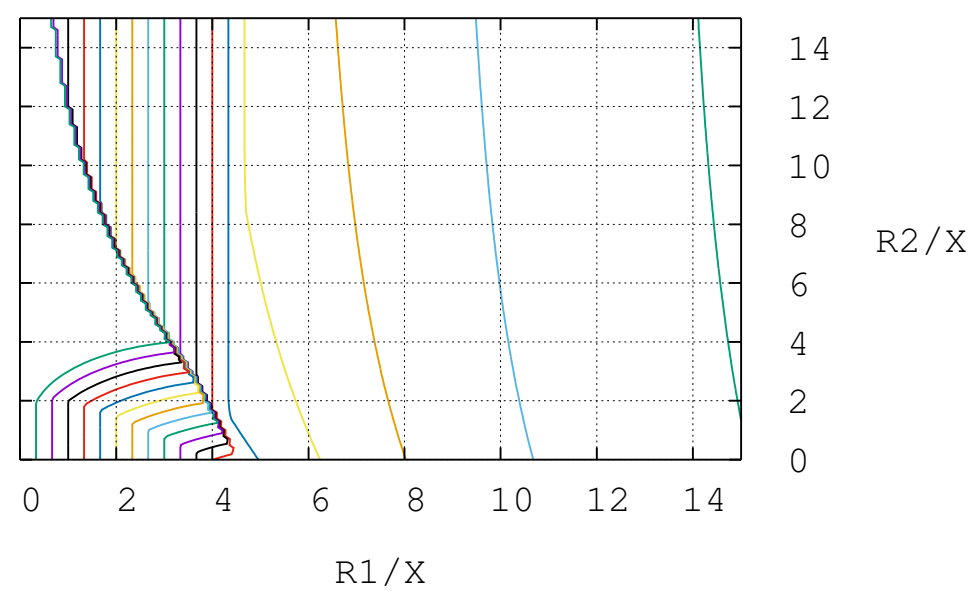

production of industry 2

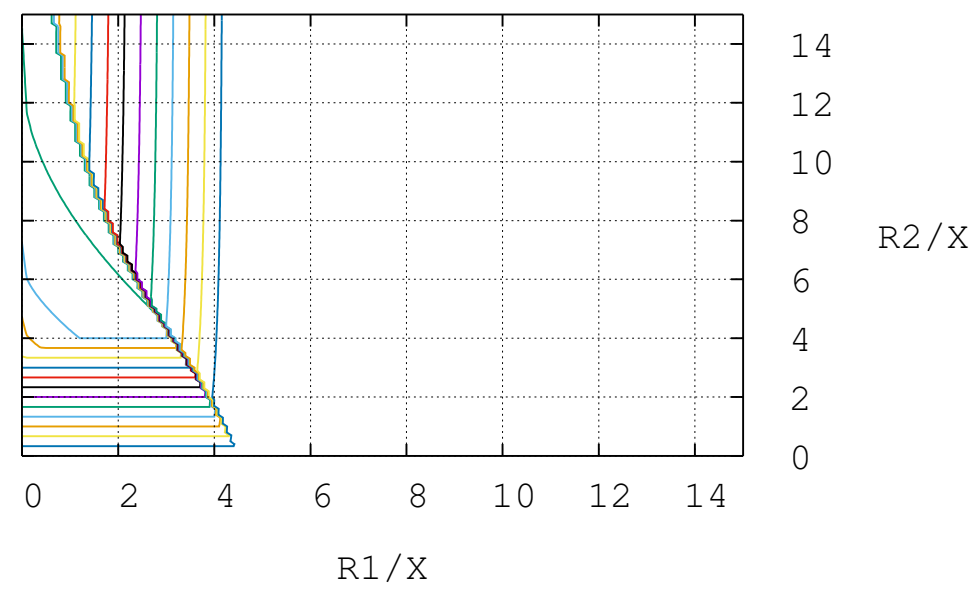

Figure 7: The contours of the productions $\tilde{Q}_{1}=\frac{Q_{1}}{X}$ and $\tilde{Q}_{2}=\frac{Q_{2}}{X}$ 


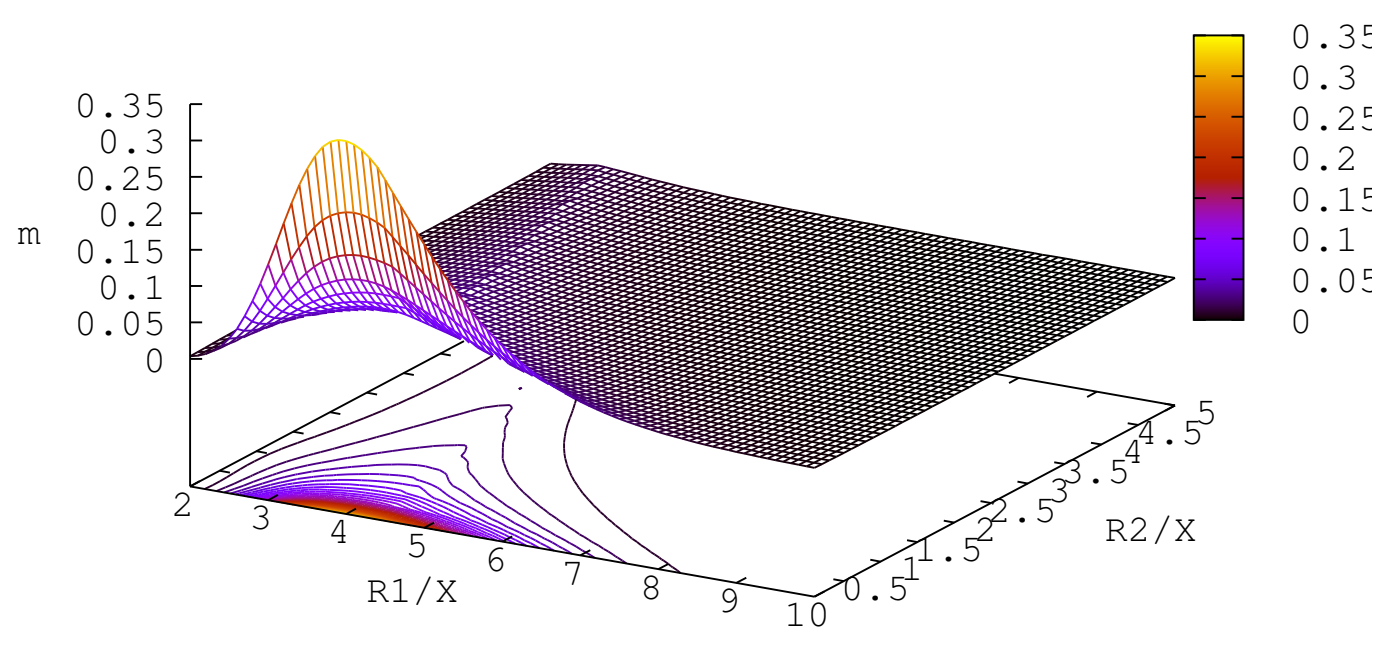

prices

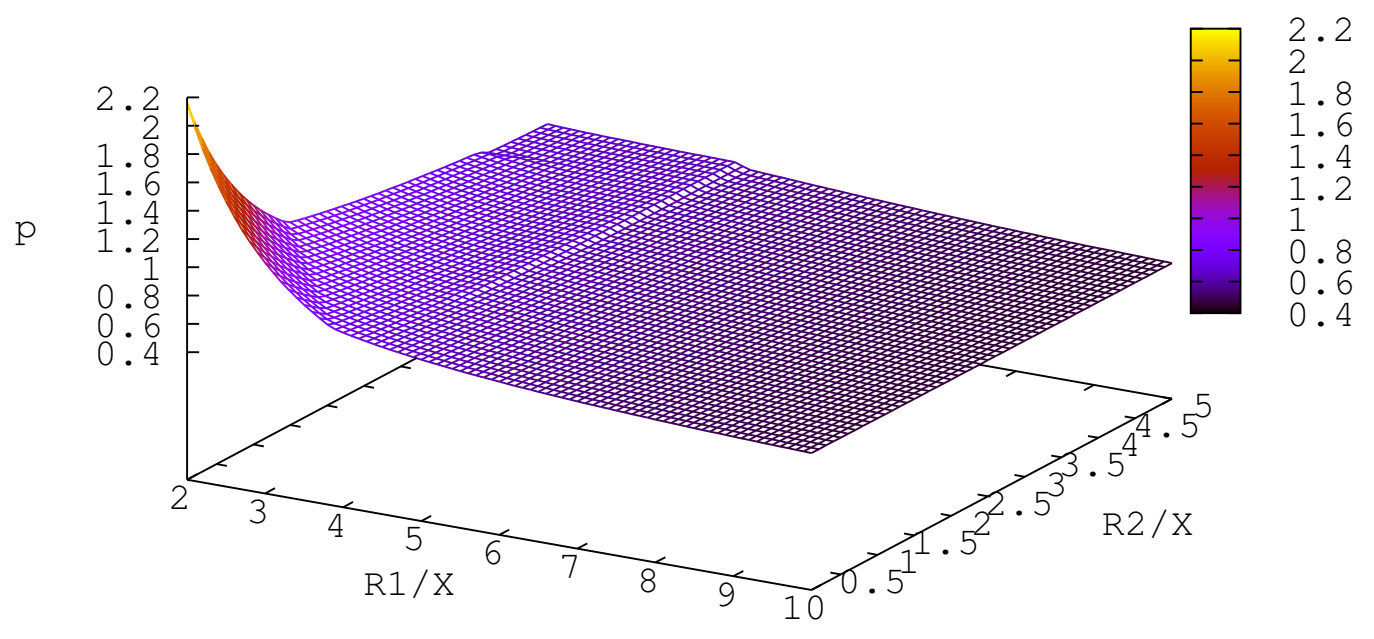

Figure 8: The distributions of the agents and the prices 


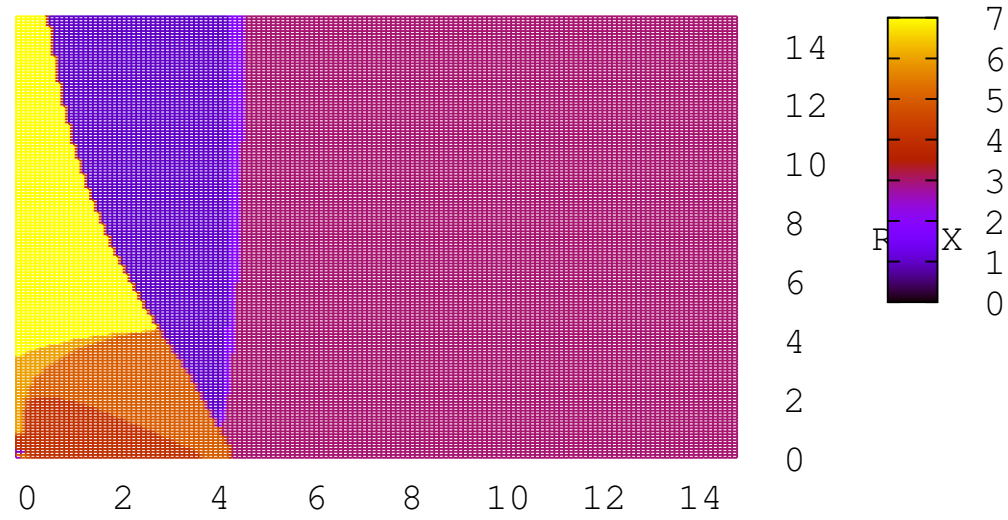

$\mathrm{R} 1 / \mathrm{X}$

Figure 9: There are seven different regimes for the Hamiltonians (see $\S 4.1$ ): all the possible regimes are present except the first regime in the case when $v_{1}+c_{1} \leq v_{2}+c_{2}$.

\subsection{Case 2}

We keep the same parameters as above except that

$$
\phi_{1}(\alpha)=0.895 \sqrt{\alpha}, \quad \phi_{2}(\alpha)=1.183 \sqrt{\alpha},
$$

In this model, the cost of production of industry 1 is smaller than that of industry 2: $c_{1}<c_{2}$, whereas the investments into prospection are more efficient for industry 2.

On Figure 10, we plot the contours of $v_{1}$ and $v_{2}$ as functions of $y_{1}=R_{1} / X$ and $y_{1}=R_{2} / X$. On Figure 11, we plot the rescaled productions $\tilde{Q}_{1}=\frac{Q_{1}}{X}$ and $\tilde{Q}_{2}=\frac{Q_{2}}{X}$ as functions of $y_{1}=R_{1} / X$ and $y_{2}=R_{2} / X$. We plot the contours of the same functions on Figure 12. The distribution of agents and the price as functions of $R_{1} / X$ and $R_{2} / X$ are plotted on Figure 13.

On Figure 14, we plot eight different zones corresponding to the different regimes of the Hamiltonian. All the eight possible regimes are present. 
contours of $\mathrm{v} 1$

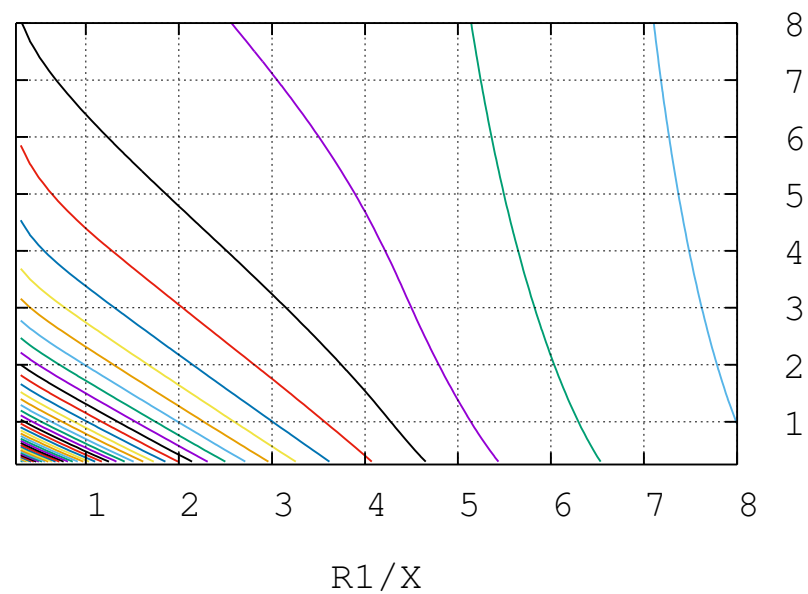

R2 / X

contours of v2

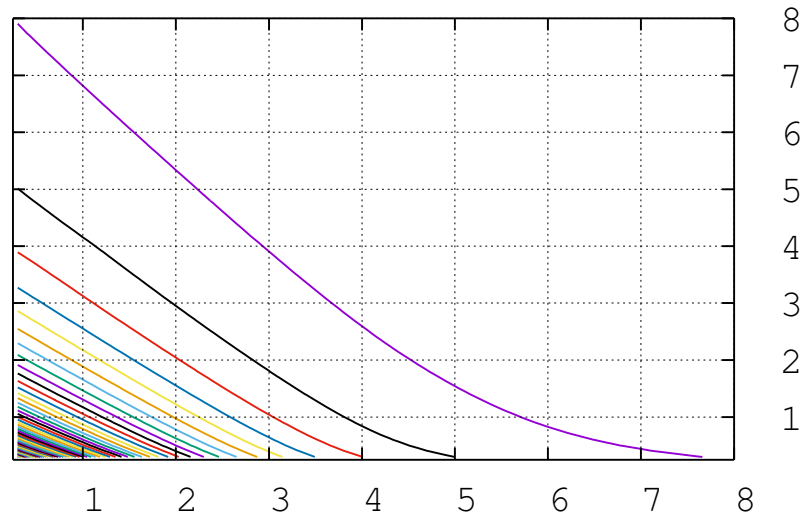

R2 / X

Figure 10: The contours of $v_{1}$ and $v_{2}$ 
production of industry 1

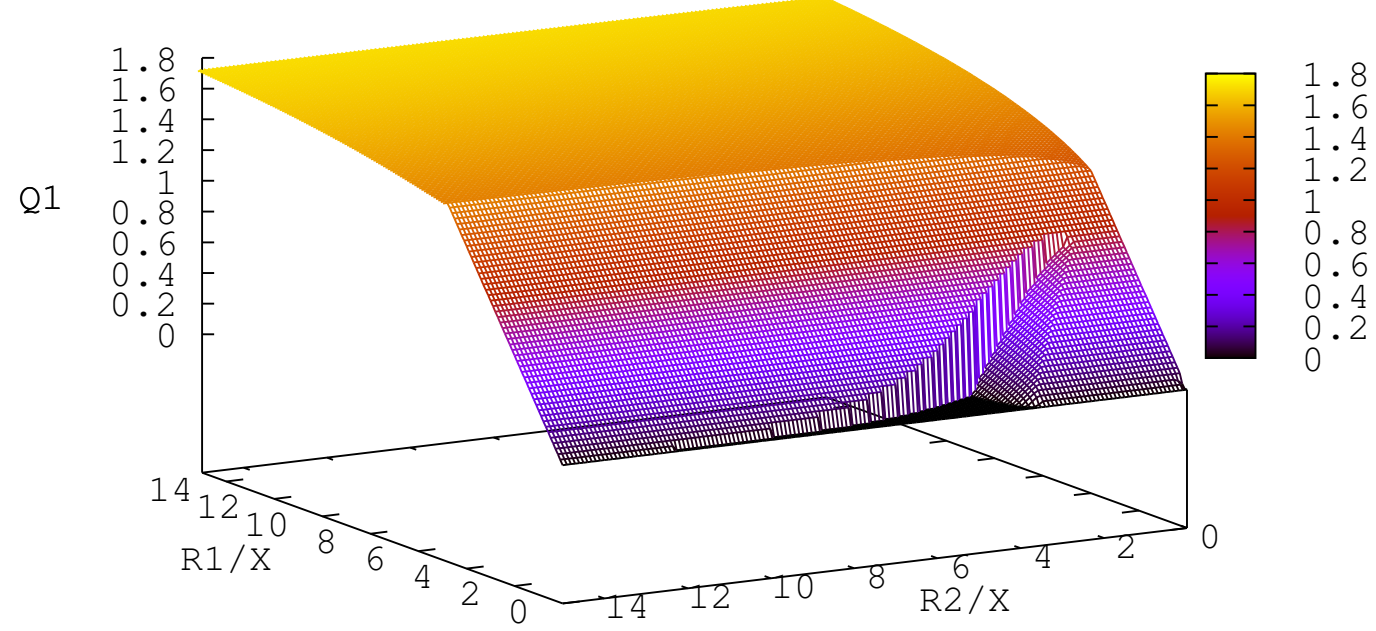

production of industry 2

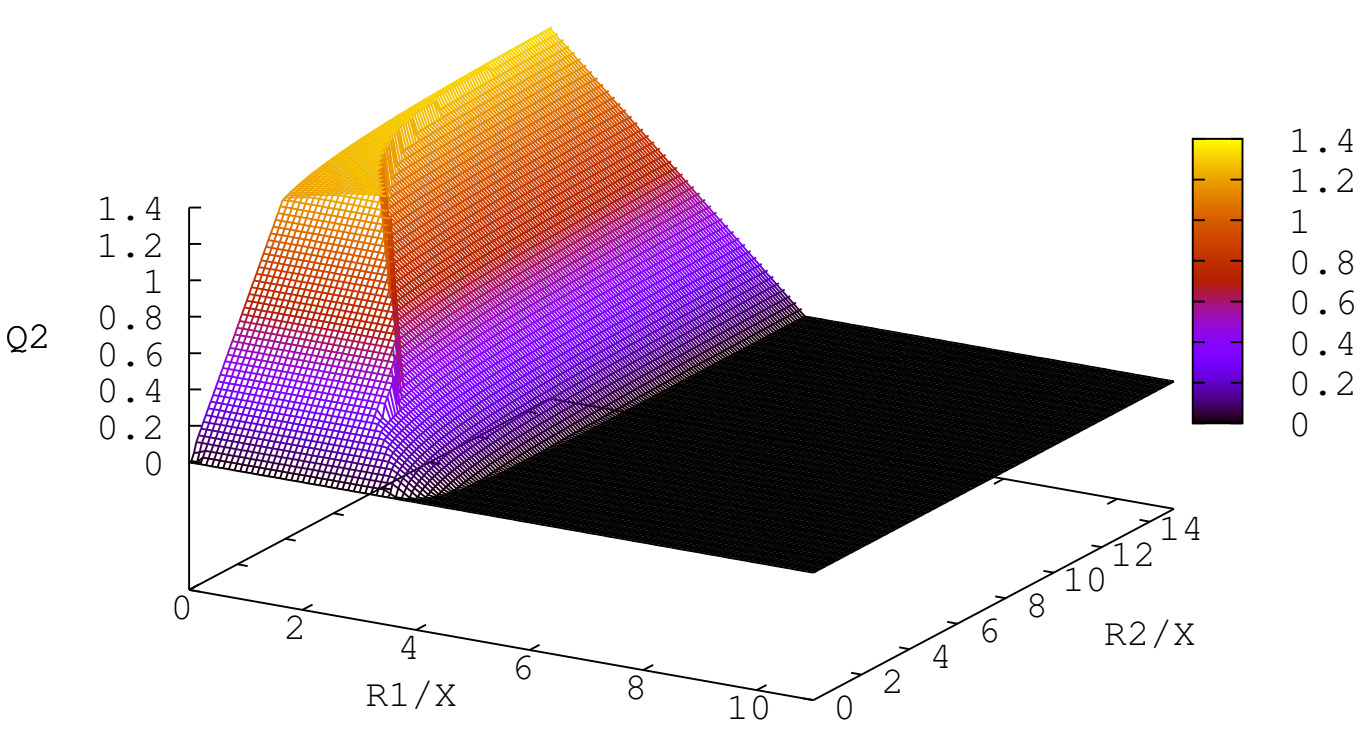

Figure 11: The productions $\tilde{Q}_{1}=\frac{Q_{1}}{X}$ and $\tilde{Q}_{2}=\frac{Q_{2}}{X}$ 
production of industry 1

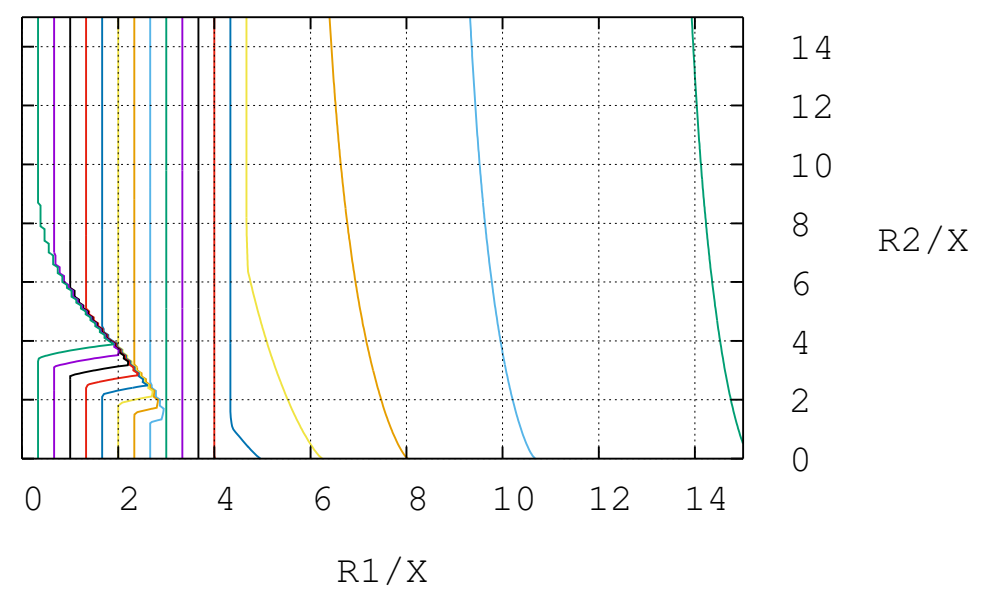

production of industry 2

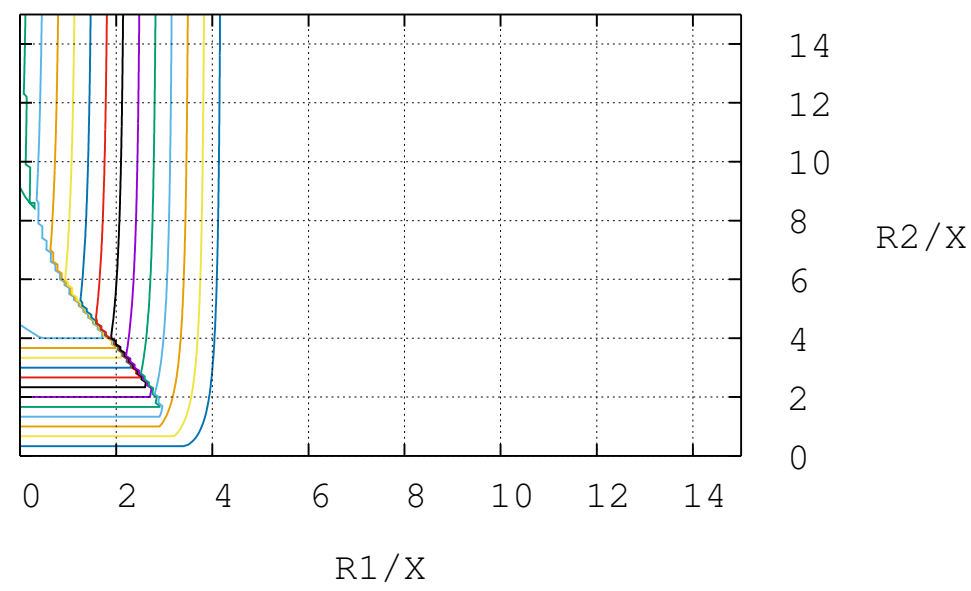

Figure 12: The contours of the productions $\tilde{Q}_{1}=\frac{Q_{1}}{X}$ and $\tilde{Q}_{2}=\frac{Q_{2}}{X}$ 


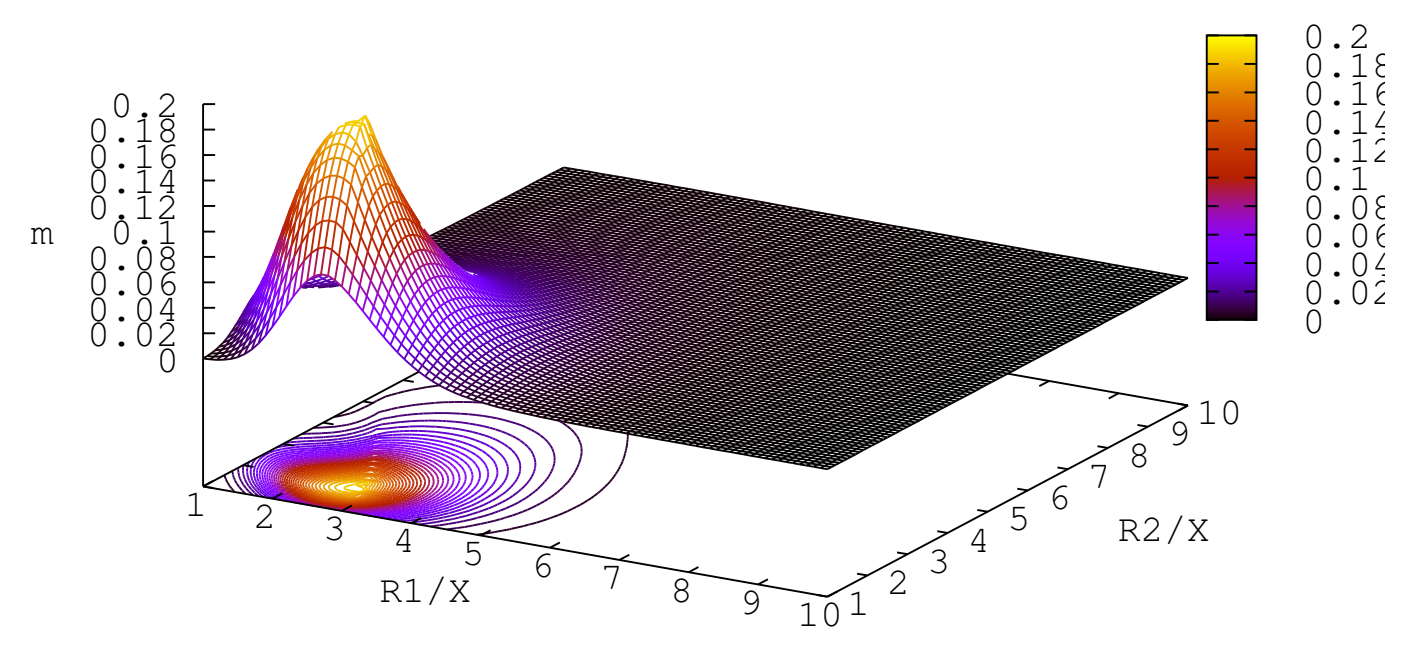

prices

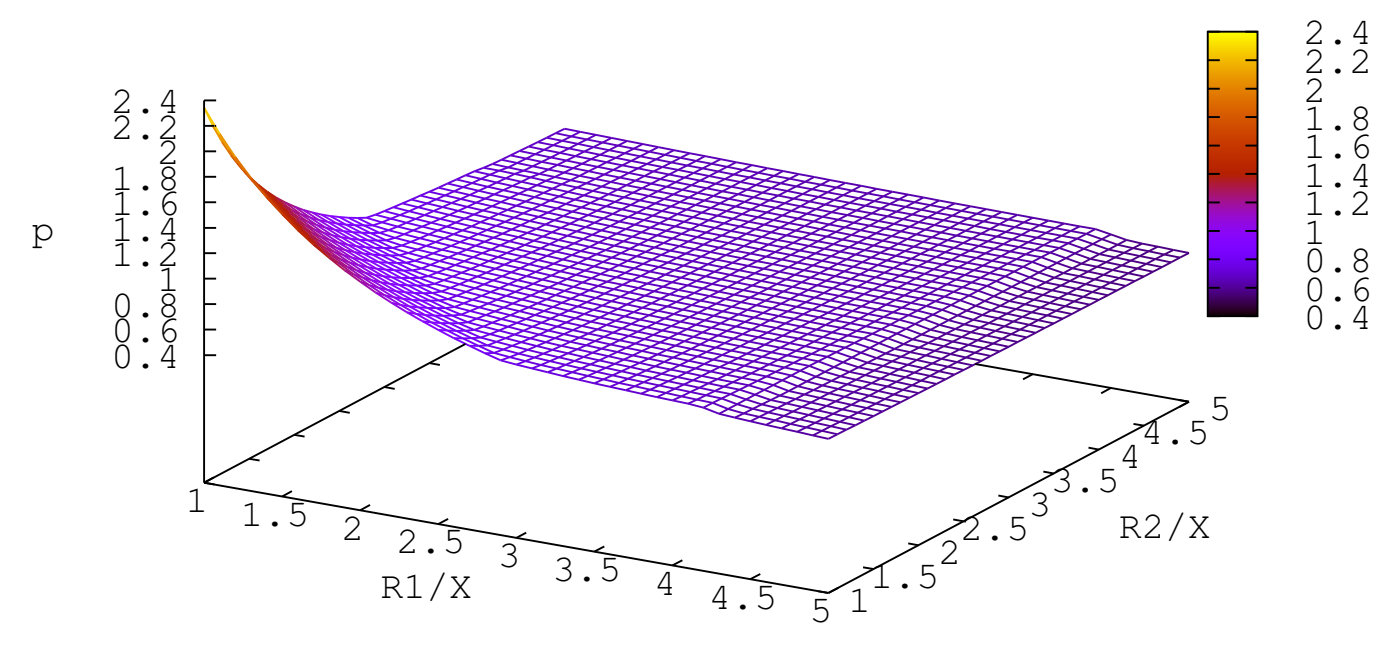

Figure 13: The distributions of the agents and the prices 


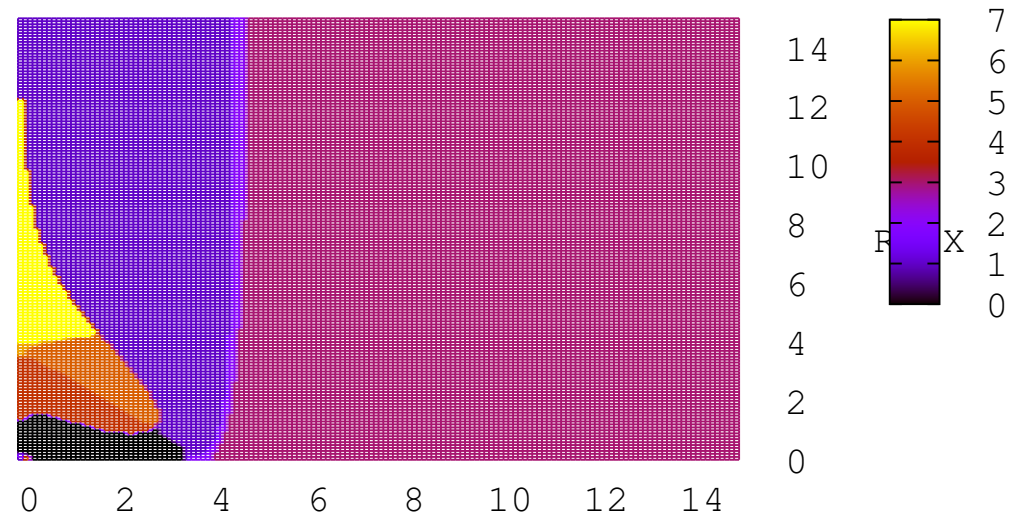

$\mathrm{R} 1 / \mathrm{X}$

Figure 14: There are eight different regimes for the Hamiltonians (see $\S 4.1$ )

\section{A A mean field games approach to Lucas-Prescott benevolent planner model}

The present mining industry model is reminiscent of the celebrated Lucas-Prescott model, see [14]: let us recall the latter and propose its interpretation in terms of mean field games.

\section{A.1 The framework}

Lucas and Prescott consider a market of production units. The size of a given unit, i.e. the capital owned by the producer is $k_{t}$. Each producer can invest in order to improve the production capacity, and therefore increase its capital: the flux of capital generated during $d t$ by an invested flux of $z_{t} d t$ is $k_{t} \Phi\left(z_{t} / k_{t}\right) d t$, where the nondecreasing and concave function $\phi$ measures the impact of the investment: therefore

$$
d k_{t}=k_{t} \Phi\left(z_{t} / k_{t}\right) d t .
$$

The price of a unit of capital is fixed by a pricing function:

$$
p_{t}=P\left(K_{t}, X_{t}\right),
$$

where $K_{t}$ is the aggregate capital and $X_{t}$ is an exogeneous parameter standing for the global state of the economy. This parameter is driven by a diffusion process:

$$
d X_{t}=\mu\left(X_{t}\right) d t+\sigma\left(X_{t}\right) d W_{t},
$$

hence it represents an aggregate risk, common to all production units. Each individual firm solves the optimal control problem: maximiz e

$$
u=\max _{z_{t} \geq 0} \mathbb{E}\left(\int_{0}^{\infty} e^{-r t}\left(p_{t} k_{t}-z_{t}\right) d t\right) .
$$




\section{A.2 The approach via mean field games}

Similarly as in $\S 2$, it is convenient to split the production units in such a way that any production unit corresponds to a unit of capital. Then the aggregate capital $K_{t}$ is the number of such production units. The value $u$ of a unit of capital can be expressed as a function of $K$ and $X$ : $u=u(K, X)$. From the dynamic programming principle, $u$ satisfies

$$
u(K, X)=(1-r d t) \max _{\alpha \geq 0} \mathbb{E}((1+\phi(\alpha) d t) u(K+d K, X+d X)+(P(K, X)-\alpha) d t) .
$$

Note the similarity with (7). A first order expansion yields

$$
\begin{aligned}
& \max _{\alpha \geq 0}(\phi(\alpha) u(K, X)-\alpha)+\partial_{K} u(K, X) \frac{d K}{d t}+\mu(X) \partial_{X} u(K, X)+\frac{\sigma^{2}(X)}{2} \partial_{X}^{2} u(K, X)+P(K, X) \\
& =r u(K, X),
\end{aligned}
$$

where the optimal control $\alpha^{*}$ is such that $\phi^{\prime}\left(\alpha^{*}\right) u(K, X)=1$. Then, at the mean field game equilibrium, $\frac{d K}{d t}=\phi\left(\alpha^{*}\right) K$. Finally, we obtain the partial differential equation:

$$
\begin{aligned}
& \max _{\alpha \geq 0}(\phi(\alpha) u(K, X)-\alpha)+K \phi\left(\alpha^{*}\right) \partial_{K} u(K, X)+\mu(X) \partial_{X} u(K, X)+\frac{\sigma^{2}(X)}{2} \partial_{X}^{2} u(K, X)+P(K, X) \\
& =r u(K, X),
\end{aligned}
$$

or in an equivalent manner

$\partial_{K}\left(K \max _{\alpha \geq 0}(\phi(\alpha) u(K, X)-\alpha)\right)+\mu(X) \partial_{X} u(K, X)+\frac{\sigma^{2}(X)}{2} \partial_{X}^{2} u(K, X)+P(K, X)=r u(K, X)$.

\section{A.3 From (64) to a Hamilton-Jacobi equation}

The aim is to find a Hamilton-Jacobi equation of the form

$$
r V(K, X)=H\left(K, X, \partial_{K} V, \partial_{X} V\right)+a(X) \partial_{X}^{2} V,
$$

in such a way that if $V$ is a solution to (65), then $u=\partial_{K} V$ is a solution to (64). Differentiating (65) with respect to $K$,

$$
\begin{aligned}
r \partial_{K} V= & \partial_{K} H\left(K, X, \partial_{K} V, \partial_{X} V\right) \\
& +\partial_{3} H\left(K, X, \partial_{K} V, \partial_{X} V\right) \partial_{K}^{2} V+\partial_{4} H\left(K, X, \partial_{K} V, \partial_{X} V\right) \partial_{K X}^{2} V+a(X) \partial_{X X K}^{3} V
\end{aligned}
$$

with self-explanatory notations. If $u=\partial_{K} V$, this yields

$$
r u=\partial_{K} H\left(K, X, u, \partial_{X} V\right)+\partial_{3} H\left(K, X, u, \partial_{X} V\right) \partial_{K} u+\partial_{4} H\left(K, X, u, \partial_{X} V\right) \partial_{X} u+a(X) \partial_{X}^{2} u
$$

Identifying, we obtain that $a(X)=\sigma^{2}(X) / 2, \partial_{4} H\left(K, X, u, \partial_{X} V\right)=\mu(X), \partial_{3} H\left(K, X, u, \partial_{X} V\right)=$ $K \Phi\left(\alpha^{*}\right)$ and $\partial_{K} H\left(K, X, u, \partial_{X} V\right)=P(K, X)+\max _{\alpha \geq 0}(\phi(\alpha) u-\alpha)$. Consider a surplus function $s(K, X)$ such that $\partial_{K} s(K, X)=P(K, X)$; we see that a good candidate for the Hamiltonian is

$$
H(K, X, u, z)=K \max _{\alpha \geq 0}(\phi(\alpha) u-\alpha)+\mu(X) z+s(K, X) .
$$


and (65) becomes

$$
r V(K, X)=K \max _{\alpha \geq 0}\left(\phi(\alpha) \partial_{K} V-\alpha\right)+\mu(X) \partial_{X} V+\frac{\sigma^{2}(X)}{2} \partial_{X}^{2} V+s(K, X),
$$

which plays the same role as (19), except that no reduced variable as been used in (66). Note that differentiating (66) with respect to $X$ and setting $w(K, X)=\partial_{X} V$ yields another partial equation:

$$
K\left(\phi\left(\alpha^{*}\right) \partial_{K} w(K, X)+\partial_{X}(\mu w)+\partial_{X}\left(\frac{\sigma^{2}}{2} \partial_{X} w\right)+\partial_{X} s(K, X)=r w(K, X) .\right.
$$

\section{A.4 Link of (66) with Lucas-Prescott benevolent planner problem}

Hamilton-Jacobi equation (66), which has been found via mean field game theory, is also satisfied by the value function of Lucas-Prescott benevolent planner problem. Indeed, Lucas-Prescott benevolent planner problem is as follows:

$$
V(K, X)=\max _{\alpha_{t}} \mathbb{E}\left(\int_{0}^{\infty} e^{-r t}\left(s\left(K_{t}, X_{t}\right)-\alpha_{t} K_{t}\right) d t\right),
$$

subject to

$$
\begin{aligned}
& d K_{t}=K_{t} \Phi\left(\alpha_{t}\right) d t, \quad K_{0}=K \\
& d X_{t}=\mu\left(X_{t}\right) d t+\sigma\left(X_{t}\right) d W_{t}, \quad X_{0}=X,
\end{aligned}
$$

and from the dynamic programming principle, $V$ solves (66).

\section{References}

[1] Y. Achdou, F.J. Buera, J-M. Lasry, P-L. Lions, and B. Moll, Partial differential equation models in macroeconomics, Philosophical Transactions of the Royal Society A: Mathematical, Physical and Engineering Sciences 372 (2014), no. 2028.

[2] Y. Achdou, J.-M. Lasry, P.-L. Lions, and J. Scheinkman, work in progress.

[3] M. Bardi and I. Capuzzo-Dolcetta, Optimal control and viscosity solutions of Hamilton-JacobiBellman equations, Systems \& Control: Foundations \& Applications, Birkhäuser Boston Inc., Boston, MA, 1997, With appendices by Maurizio Falcone and Pierpaolo Soravia. MR 1484411 (99e:49001)

[4] I. Capuzzo-Dolcetta and P.-L. Lions, Hamilton-Jacobi equations with state constraints, Trans. Amer. Math. Soc. 318 (1990), no. 2, 643-683. MR 951880 (90g:49021)

[5] P. Cardaliaguet, F. Delarue, J-M. Lasry, and P-L. Lions, The master equation and the convergence problem in mean field games, arXiv preprint arXiv:1509.02505 (2015).

[6] O. Guéant, J-M. Lasry, and P-L. Lions, Mean field games and applications, Paris-Princeton Lectures on Mathematical Finance 2010, Lecture Notes in Math., vol. 2003, Springer, Berlin, 2011, pp. 205266. MR 2762362

[7] P. Krusell and A. A. Smith, Income and wealth heterogeneity in the macroeconomy, Journal of Political Economy 106 (1998), no. 5, 867-896.

[8] A. Lachapelle, J-M. Lasry, C-A. Lehalle, and P-L. Lions, Efficiency of the price formation process in presence of high frequency participants: a mean field game analysis, Mathematics and Financial Economics (2013), 1-40. 
[9] J.-M. Lasry and P.-L. Lions, Nonlinear elliptic equations with singular boundary conditions and stochastic control with state constraints. I. The model problem, Math. Ann. 283 (1989), no. 4, 583630. MR 990591

[10] J-M. Lasry and P-L. Lions, Jeux à champ moyen. I. Le cas stationnaire, C. R. Math. Acad. Sci. Paris 343 (2006), no. 9, 619-625. MR MR2269875 (2007m:91021)

[11] _ Jeux à champ moyen. II. Horizon fini et contrôle optimal, C. R. Math. Acad. Sci. Paris 343 (2006), no. 10, 679-684. MR MR2271747 (2007m:91022)

[12] _ Mean field games, Jpn. J. Math. 2 (2007), no. 1, 229-260. MR MR2295621

[13] P.L Lions, Generalized solutions of Hamilton-Jacobi equations, Research Notes in Mathematics 69, Pitman, Boston, 1982.

[14] R. E. Lucas, Jr. and E. C. Prescott, Investment under uncertainty, Econometrica 39 (1971), 659-681. MR 0398471

[15] H. M. Soner, Optimal control with state-space constraint. I, SIAM J. Control Optim. 24 (1986), no. 3, 552-561. MR MR838056 (87e:49029)

[16] Optimal control with state-space constraint. II, SIAM J. Control Optim. 24 (1986), no. 6, 1110-1122. MR MR861089 (87k:49021) 